International Electronic Journal of Algebra

Volume 27 (2020) 13-42

DOI: $10.24330 /$ ieja.662946

\title{
ON A SPECIAL PRESENTATION OF MATRIX ALGEBRAS
}

\author{
Geir Agnarsson and Samuel S. Mendelson \\ Received: 7 June 2018; Revised: 25 June 2019; Accepted: 29 June 2019 \\ Communicated by Abdullah Harmancı
}

\begin{abstract}
Recognizing when a ring is a complete matrix ring is of significant importance in algebra. It is well-known folklore that a ring $R$ is a complete $n \times n$ matrix ring, so $R \cong M_{n}(S)$ for some ring $S$, if and only if it contains a set of $n \times n$ matrix units $\left\{e_{i j}\right\}_{i, j=1}^{n}$. A more recent and less known result states that a ring $R$ is a complete $(m+n) \times(m+n)$ matrix ring if and only if, $R$ contains three elements, $a, b$, and $f$, satisfying the two relations $a f^{m}+f^{n} b=1$ and $f^{m+n}=0$. In many instances the two elements $a$ and $b$ can be replaced by appropriate powers $a^{i}$ and $a^{j}$ of a single element $a$ respectively. In general very little is known about the structure of the ring $S$. In this article we study in depth the case $m=n=1$ when $R \cong M_{2}(S)$. More specifically we study the universal algebra over a commutative ring $A$ with elements $x$ and $y$ that satisfy the relations $x^{i} y+y x^{j}=1$ and $y^{2}=0$. We describe completely the structure of these $A$-algebras and their underlying rings when $\operatorname{gcd}(i, j)=1$. Finally we obtain results that fully determine when there are surjections onto $M_{2}(\mathbb{F})$ when $\mathbb{F}$ is a base field $\mathbb{Q}$ or $\mathbb{Z}_{p}$ for a prime number $p$.
\end{abstract}

Mathematics Subject Classification (2010): 15B33, 16S15, 16S50

Keywords: Matrix ring, matrix algebra, finite presentation

\section{Introduction}

1.1. History and motivation. Matrix rings and algebras have been studied for a long time. For examples of their importance and study see [7, Chapter 7$]$ and $[10$, Chapter 1,6$]$. We say that a unital ring $R$ is a complete $n \times n$ matrix ring over a ring $S$ if $R \cong M_{n}(S)$. Recognizing a complete matrix ring, or algebra, is however not obvious. Recall that $n \times n$ matrix units in a unital ring $R$ is a set of elements $\left\{e_{i j}: 1 \leq i, j \leq n\right\} \subseteq R$ that satisfy

$$
\sum_{i=1}^{n} e_{i i}=1_{R} \text { and } e_{i j} e_{k \ell}=\delta_{j k} e_{i \ell},
$$

where $\delta_{j \ell}$ is the Kronecker delta function [10, Chapter 1]. The most well-known element-wise characterization of a complete $n \times n$ matrix ring is given by the following folklore theorem [10, Prop. 11.3, p. 22]. 
Theorem 1.1. A unital ring $R$ is a complete $n \times n$ matrix ring over some ring $S$, that is $R \cong M_{n}(S)$, if and only if it contains a set of $n \times n$ matrix units.

The ring $S$ in the above Theorem 1.1 is completely determined by

$$
S=\left\{\sum_{i=1}^{n} e_{i 1} x e_{1 i} ; x \in R\right\} .
$$

However, these matrix units can be difficult to find and tedious to verify. In 1990, Chatters in [5] posed the following question: Let $\mathbb{H}$ be the integer quaternions and $T(n)=\left(\mathbb{\mathbb { H }} \frac{n \mathbb{H}}{\mathbb{H}}\right)$. For which, if any, values of $n$ is the tiled matrix ring $T(n)$ a complete matrix ring? At first glance, $T(n)$ does not appear to be a complete matrix ring. However, using properties of $\mathbb{H}$ and finding suitable matrix units, it turns out that $T(n) \cong M_{2}(S)$ for some $S$ (not necessarily unique) for odd values of $n[9]$.

In 1996, Agnarsson, Amitsur, and Robson in [2] refined structural results from [9] and obtained the following two theorems, the first of which is a three-element relations.

Theorem $1.2([2])$. A ring $R$ is a complete $(m+n) \times(m+n)$ matrix ring $M_{m+n}(S)$ if and only if it contains elements $a, b$, and $f$ satisfying the relations $a f^{m}+f^{n} b=$ 1 and $f^{m+n}=0$.

Using this result they investigated rings of differential operators [2].

In 1996, Lam and Leroy in [8] investigated relations for recognizing matrix rings, in particular these three-element relations. Using the above Theorem 1.2 from [2] they give an eigenring description, using a certain nilpotent element in $R$, for the ring $S$ over which $R$ is a complete $(m+n) \times(m+n)$ matrix ring. In addition, they use Theorem 1.2 to study Ore extension rings (or skew-polynomial rings).

Under these relations however, very little is known about the explicit structure of the ring $S$. In fact, under certain circumstances, $S$ may be the trivial ring. Their next result is on two-element relations.

Theorem $1.3([2])$. A ring $R$ is a complete $(m+n) \times(m+n)$ matrix ring $M_{m+n}(S)$ if and only if it contains elements $a$ and $f$ satisfying the relations $a^{m} f^{m}+f^{n} a^{n}=$ 1 and $f^{m+n}=0$.

Note that the characterizations given in Theorem 1.1 uses $n^{2}$ elements together with $n^{4}+1$ relations among them to characterize a complete $n \times n$ matrix ring, whereas Theorems 1.2 and 1.3 use three and two elements respectively and two relations involving these elements to characterize complete $(m+n) \times(m+n)$ matrix 
rings. In particular, the number or elements and relations in Theorems 1.2 and 1.3 are not functions of the size of the matrix ring. These are the only known such characterizations for complete matrix rings.

Under the two-element relations in Theorem 1.3, it is easy to find matrices over $S$ that satisfy the two relations: $a$ can be the matrix with 1 's along its subdiagonal and 0's everywhere else, while $f$ can be the matrix with 1's along its super-diagonal and 0's everywhere else. It is therefore natural to ask what happens if the first relation in Theorem 1.2 is replaced by $a^{i} f^{m}+f^{n} a^{j}=1$. The ring $R$ is by Theorem 1.2 a complete $(m+n) \times(m+n)$ matrix ring, but it could be the trivial ring; in [1] it is shown that if a ring $R$ contains elements $a$ and $b$ such that $a b^{m}+b^{n} a=1$ and $b^{m+n}=0$ where $m \neq n$, then $R$ is the trivial ring. This result together with Theorem 1.3 strongly suggest the study of the universal ring that contains two elements $a$ and $f$ that satisfy $a^{i} f^{m}+f^{n} a^{j}=1$ and $f^{m+n}=0$. This is the motivation for this article.

1.2. Basic setup and definitions. The set $\{1,2,3, \ldots\}$ of natural numbers will be denoted by $\mathbb{N}$ and for $n \in \mathbb{N}$ we let $[n]=\{1,2, \ldots, n\}$. The field of rational numbers will be denoted by $\mathbb{Q}$ and for a prime $p$, the unique finite field with $p$ elements will be denoted by $\mathbb{Z}_{p}$. For the rest of this article, all rings will be associative and unital, that is with a multiplicative unit 1, and all homomorphisms will be assumed unital. We begin with some definitions.

Definition 1.4. Let $A$ be a commutative ring.

(I) The free monoid $\left\langle x_{1}, \ldots, x_{n}\right\rangle$ on $n$ indeterminates is the set of words made by the indeterminates $x_{i}$ along with the binary operation of concatenation of words with identity, the empty word, which we denote by 1.

(II) The free $A$-algebra $A\left\langle x_{1}, \ldots, x_{n}\right\rangle$ over the ring $A$ on $n$ indeterminates is the set of formal linear combinations over $A$ of elements from $\left\langle x_{1}, \ldots, x_{n}\right\rangle$. Addition is defined as formal sums of elements and multiplication is defined as concatenation of basis elements extended as an $A$-bilinear operation.

(III) For a subset $\left\{f_{1}, \ldots, f_{N}\right\} \subseteq A\left\langle x_{1}, \ldots, x_{n}\right\rangle$, the free $A$-algebra on $x_{1}, \ldots, x_{n}$ satisfying $f_{1}=\cdots=f_{n}=0$, denoted by $A\left\langle x_{1}, \ldots, x_{n}: f_{1}, \ldots, f_{N}\right\rangle$, is the quotient $A$-algebra $A\left\langle x_{1}, \ldots, x_{n}\right\rangle / I$ where $I=\left(f_{1}, \ldots, f_{N}\right)$ is the two-sided ideal of $A\left\langle x_{1}, \ldots, x_{n}\right\rangle$ generated by $f_{1}, \ldots, f_{N}$.

We are interested in rings with two elements, $x$ and $y$, satisfying the relations $x^{i} y^{m}+y^{n} x^{j}=1$ and $y^{m+n}=0$. In this article we will investigate the free object satisfying these two relations. 
Definition 1.5. For a commutative ring $A$ and natural numbers $i, j, m, n \in \mathbb{N}$, let $R(A ; i, j, m, n):=A\left\langle x, y: x^{i} y^{m}+y^{n} x^{j}=1, y^{m+n}=0\right\rangle$.

By Theorem 1.2 we have $R(A ; i, j, m, n) \cong M_{m+n}(S)$ for some $A$-algebra $S$. As we have very little information about $S$, we introduce the following sets similarly as in [1].

Definition 1.6. For a commutative ring $A$ define the sets $\mathcal{A}_{A}, \mathcal{B}_{A}, \mathcal{C}_{A} \subseteq \mathbb{N}^{4}$ as follows:

$\mathcal{A}_{A}$ : the set of $(i, j, m, n) \in \mathbb{N}^{4}$ such that there is a non-trivial homomorphism $R(A ; i, j, m, n) \rightarrow M_{m+n}(A)$.

$\mathcal{B}_{A}$ : the set of $(i, j, m, n) \in \mathbb{N}^{4}$ such that there is a non-trivial homomorphism $R(A ; i, j, m, n) \rightarrow M_{N}(A)$ for some $N \in \mathbb{N}$.

$\mathcal{C}_{A}$ : the set of $(i, j, m, n) \in \mathbb{N}^{4}$ with $R(A ; i, j, n, m)$ non-trivial.

Remark 1.7. Here $\mathcal{A}_{A}$ is the set of those $(i, j, m, n) \in \mathbb{N}^{4}$ such that there exist $(m+n) \times(m+n)$ matrices $x$ and $y$ over $A$ satisfying the defining relations for $R(A ; i, j, m, n), \mathcal{B}_{A}$ is the set of those $(i, j, m, n) \in \mathbb{N}^{4}$ such that there exist some finite rank matrices $x$ and $y$ over $A$ satisfying the defining relations for $R(A ; i, j, m, n)$, lastly, $\mathcal{C}_{A}$ is here the set of those $(i, j, m, n) \in \mathbb{N}^{4}$ such that there exist "infinite" matrices $x$ and $y$ over $A$ satisfying the defining relations for $R(A ; i, j, m, n)$.

A big motivating question is as follows:

Question 1.8. For a given commutative ring $A$, can we describe each of the sets $\mathcal{A}_{A}, \mathcal{B}_{A}$ and $\mathcal{C}_{A}$ from Definition 1.6?

The purpose of this article is in part to extend the results in [1] that partly answer the above Question 1.8. The article is organized as follows.

In Section 2 we will derive numerous structural properties of $R(A ; i, j, 1,1) \cong$ $M_{2}(S)$ and completely describe the ring $S$ for relatively prime integers $i$ and $j$.

In Section 3 we investigate $\mathcal{A}_{\mathbb{F}}$ when when $m=n=1$ and $\mathbb{F}$ is one of the base fields $\mathbb{Q}$ or $\mathbb{Z}_{p}$ where $p$ is a prime number. We will determine all $i$ and $j$ such that $(i, j, 1,1) \in \mathcal{A}_{\mathbb{F}}$ for these base fields, that is, we determine when exactly there are $2 \times 2$ matrices over $\mathbb{F} \in\left\{\mathbb{Q}, \mathbb{Z}_{p}\right\}$ that satisfy the defining relations for $R(\mathbb{F} ; i, j, 1,1)$ from Definition 1.5.

\section{The case $m=n=1$}

In this section, we will give an explicit description of $R(A ; i, j, m, n)$ from Definition 1.5 when $\operatorname{gcd}(i, j)=1$ and $m=n=1$. 
2.1. Relations and reductions. In this subsection we will derive some technical results for $R(A ; i, j, 1,1)$.

Lemma 2.1. For the generators $x, y \in R(A ; i, j, 1,1)$, we have the following relations:

$$
y x^{i} y=y x^{j} y=y, \quad y x^{i+j} y=0, \quad y x^{2 i} y=-y x^{2 j} y .
$$

Proof. We have that $x^{i} y+y x^{j}=1$ and $y^{2}=0$ and hence

$$
y=y \cdot 1=y\left(x^{i} y+y x^{j}\right)=y x^{i} y+y^{2} x^{j}=y x^{i} y .
$$

Similarly we obtain $y=\left(x^{i} y+y x^{j}\right) y=y x^{j} y$.

Using the above we get

$$
y x^{i+j} y=y x^{j} x^{i} y=y x^{j}\left(x^{i} y+y x^{j}\right) x^{i} y=y x^{i+j} y x^{i} y+y x^{j} y x^{i+j} y=2 y x^{i+j} y,
$$

and so $y x^{i+j} y=0$.

Finally, using the last two results we get

$0=y x^{i+j} y=y x^{i} x^{j} y=y x^{i}\left(x^{i} y+y x^{j}\right) x^{j} y=y x^{2 i} y x^{j} y+x^{i} y x^{2 j} y=y x^{2 i} y+y x^{2 j} y$, and thus $y x^{2 i} y=-y x^{2 j} y$.

We can now prove our first theorem.

Theorem 2.2. $R(k ; i, j, 1,1)=R(k ; j, i, 1,1)$.

Proof. Using Lemma 2.1, $x^{i} y+y x^{j}=1$ and $y^{2}=0$, we get

$$
\begin{aligned}
x^{j} y+y x^{i} & =\left(x^{i} y+y x^{j}\right)\left(x^{j} y+y x^{i}\right) \\
& =x^{i}\left(y x^{j} y\right)+x^{i} y^{2} x^{j}+y x^{2 j} y+y x^{j} y x^{i} \\
& =x^{i} y+y x^{2 j} y+y x^{i},
\end{aligned}
$$

and so $y x^{2 j} y=x^{j} y-x^{i} y$. Similarly, expanding $\left(x^{j} y+y x^{i}\right)\left(x^{i} y+y x^{j}\right)$, we get $y x^{2 i} y=y x^{i}-y x^{j}$. By Lemma 2.1 again, we know that $y x^{2 i} y=-y x^{2 j} y$, so $x^{j} y+y x^{i}=x^{i} y+y x^{j}=1$, thus $R(A ; i, j, 1,1) \subseteq R(A ; j, i, 1,1)$. By symmetry, we see $x^{j} y+y x^{i}=1$ and $y^{2}=0$ implies $x^{i} y+y x^{j}=1$ in $R(A ; j, i, 1,1)$ and so $x^{i} y+y x^{j}=1$, thus $R(A ; j, i, 1,1) \subseteq R(A ; i, j, 1,1)$, and hence $R(A ; i, j, 1,1)=R(A ; j, i, 1,1)$.

Note that Theorem 2.2 is stronger than it appears; it is clear that $R(A ; i, j, 1,1)$ is anti-isomorphic to $R(A ; j, i, 1,1)$. However, Theorem 2.2 states the rings are actually equal as sets. Without loss of generality, we can therefore assume either $i \leq j$ or $j \leq i$. For the rest of this section we will assume the latter. 
Lemma 2.3. For an arbitrary $n \in \mathbb{N}$ and the generators $x, y \in R(A ; i, j, 1,1)$ we have:

$$
\begin{aligned}
& y x^{i n}=(-1)^{n} x^{j n} y+\sum_{k=0}^{n-1}(-1)^{n-1-k} x^{(n-1) j+k(i-j)} \\
& y x^{j n}=(-1)^{n} x^{i n} y+\sum_{k=0}^{n-1}(-1)^{k} x^{(n-1) j+k(i-j)} .
\end{aligned}
$$

Proof. Since $y x^{i}=1-x^{j} y$ and $y x^{j}=1-x^{i} y$ by Theorem 2.2, we clearly have (1) and (2) for $n=1$. We proceed by induction on $n$ and assume (1) to hold for $n$. In that case we get

$$
\begin{aligned}
y x^{i(n+1)} & =\left(y x^{i n}\right) x^{i} \\
& =(-1)^{n} x^{j n} y x^{i}+\sum_{k=0}^{n-1}(-1)^{n-1-k} x^{(n-1) j+k(i-j)+i} \\
& =(-1)^{n} x^{j n}\left(1-x^{j} y\right)+\sum_{k=0}^{n-1}(-1)^{n-(k+1)} x^{n j+(k+1)(i-j)} \\
& =(-1)^{n} x^{j n}+(-1)^{n+1} x^{j(n+1)} y+\sum_{k=1}^{n}(-1)^{n-k} x^{n j+k(i-j)} \\
& =(-1)^{n+1} x^{j(n+1)} y+\sum_{k=0}^{n}(-1)^{n-k} x^{n j+k(i-j)} .
\end{aligned}
$$

Thus, for every $n \in \mathbb{N}$ we have (1). In exactly the same way we can prove (2) by using $y x^{i}=1-x^{j} y$ in the inductive step. Hence, by induction we have (1) and (2) for every $n \in \mathbb{N}$.

Lemma 2.4. Let $p(x), q(x) \in A[x]$. If $p(x) y=q(x) y$ in $R(A ; i, j, 1,1)$, then $p(x)=$ $q(x)$.

Proof. Suppose $p(x) y=q(x) y$ for some polynomials $p(x), q(x) \in A[x]$. Then

$$
p(x) y x^{j}=p(x)\left(1-x^{i} y\right)=p(x)-p(x) x^{i} y=p(x)-x^{i} p(x) y,
$$

since $p(x)$ is a polynomial in $x$ and thus commutes with $x^{i}$. By the same argument we have $q(x) y x^{j}=q(x)-x^{i} q(x) y$. Since $p(x) y=q(x) y$, we get

$$
p(x)-x^{i} p(x) y=p(x) y x^{j}=q(x) y x^{j}=q(x)-x^{i} q(x) y=q(x)-x^{i} p(x) y
$$

and hence $p(x)=q(x)$.

The next lemma will come in handy later on.

Lemma 2.5. If $i \neq j$, then $x$ is invertible in $R(A ; i, j, 1,1)$. 
Proof. By Theorem 2.2 we may without loss of generality assume $i>j$. We then get

$$
\begin{aligned}
1 & =x^{i} y+y x^{j} \\
& =x^{i-j}\left(x^{j} y\right)+y x^{j} \\
& =x^{i-j}\left(1-y x^{i}\right)+y x^{j} \\
& =\left(x^{i-j-1}-x^{i-j} y x^{i-1}+y x^{j-1}\right) x .
\end{aligned}
$$

Similarly, we can show $1=x\left(x^{j-1} y+x^{i-j-1}-x^{i-1} y x^{i-j}\right)$, and so $x$ is invertible.

We are now able to show a useful relation for $x$.

Theorem 2.6. If $i>j$ and $\operatorname{gcd}(i, j)=d$, then in $R(A ; i, j, 1,1)$ we have

$$
x^{((i+j) / d-1)(i-j)}=\sum_{k=1}^{(i+j) / d-1}(-1)^{k+1} x^{((i+j) / d-1-k)(i-j)} .
$$

Proof. We will evaluate the element $y x^{(i j) / d} y$ in two ways using our relations for $y x^{i n}$ and $y x^{j n}$. Here, let $a=i / d$ and $b=j / d$. By Lemma 2.3 we have for $n=b$ and $n=a$ respectively that

$$
y x^{(i j) / d} y=\sum_{k=0}^{b-1}(-1)^{b-1-k} x^{(b-1) j+k(i-j)} y \text { and } y x^{(i j) / d} y=\sum_{k=0}^{a-1}(-1)^{k} x^{(a-1) j+k(i-j)} y,
$$

and hence

$$
\sum_{k=0}^{a-1}(-1)^{k} x^{(a-1) j+k(i-j)} y=\sum_{k=0}^{b-1}(-1)^{b-1-k} x^{(b-1) j+k(i-j)} y .
$$

Using Lemma 2.4 we get

$$
0=\sum_{k=0}^{b-1}(-1)^{b-k} x^{(b-1) j+k(i-j)}+\sum_{k=0}^{a-1}(-1)^{k} x^{(a-1) j+k(i-j)} .
$$

Since $a j=b i$ we have, $(b-1) j+k(i-j)=(a-1) j+(k-b)(i-j)$, and so

$$
\sum_{k=0}^{a-1}(-1)^{k} x^{(a-1) j+k(i-j)}=\sum_{k=b}^{a+b-1}(-1)^{b-k} x^{(b-1) j+k(i-j)},
$$

and therefore

$$
\begin{aligned}
0 & =\sum_{k=0}^{b-1}(-1)^{b-k} x^{(b-1) j+k(i-j)}+\sum_{k=b}^{a+b-1}(-1)^{b-k} x^{(b-1) j+k(i-j)} \\
& =\sum_{k=0}^{a+b-1}(-1)^{b-k} x^{(b-1) j+k(i-j)}
\end{aligned}
$$


Since $x$ is invertible by Theorem 2.5 , we obtain

$$
0=\sum_{k=0}^{a+b-1}(-1)^{b-k} x^{k(i-j)}
$$

and by re-indexing and shifting the last term we have,

$$
x^{(a+b-1)(i-j)}=\sum_{k=1}^{a+b-1}(-1)^{k+1} x^{(a+b-1-k)(i-j)} .
$$

Writing out the above sum, we see that the relation in Theorem 2.6 gives an alternating series relation for $x^{(i+j) /(d-1)(i-j)}$. Letting $m=(i+j) / d$ we have

$$
x^{(m-1)(i-j)}=x^{(m-2)(i-j)}-x^{(m-3)(i-j)}+\cdots+(-1)^{(i+j) / d} .
$$

Theorem 2.6 can be used to obtain the following.

Corollary 2.7. If $d=\operatorname{gcd}(i, j)$, then $x^{\left(i^{2}-j^{2}\right) / d}=(-1)^{(i+j) / d}$.

Proof. By Theorem 2.6, we have

$$
x^{((i+j) / d-1)(i-j)}=\sum_{k=1}^{(i+j) / d-1}(-1)^{k+1} x^{((i+j) / d-1-k)(i-j)} .
$$

Multiplying both sides of this relation by $x^{i-j}$ and using Theorem 2.6 again, we get

$$
\begin{aligned}
x^{(i+j)(i-j) / d} & =\sum_{k=1}^{(i+j) / d-1}(-1)^{k+1} x^{((i+j) / d-k)(i-j)} \\
& =x^{((i+j) / d-1)(i-j)}+\sum_{k=2}^{(i+j) / d-1}(-1)^{k+1} x^{((i+j) / d-k)(i-j)} \\
& =\sum_{k=1}^{(i+j) / d-1}(-1)^{k+1} x^{((i+j) / d-k-1)(i-j)} \\
& -\sum_{k=1}^{(i+j) / d-2}(-1)^{k+1} x^{((i+j) / d-k-1)(i-j)} \\
& (-1)^{(i+j) / d} .
\end{aligned}
$$

If $i \neq j$, Corollary 2.7 shows that $x$ can be viewed as a root of unity.

Proposition 2.8. The elements $x^{i+j}$ and $x^{i}-x^{j}$ are in the center of $R(A ; i, j, 1,1)$. 
Proof. It suffices to show that $x^{i+j}$ and $x^{i}-x^{j}$ commute with $y$. By definition of $R(A ; i, j, 1,1)$ we get

$$
y\left(x^{i+j}\right)=\left(y x^{i}\right) x^{j}=\left(1-x^{j} y\right) x^{j}=x^{j}-x^{j} y x^{j}=x^{j}-x^{j}\left(1-x^{i} y\right)=\left(x^{i+j}\right) y,
$$

and

$$
y\left(x^{i}-x^{j}\right)=y x^{i}-y x^{j}=\left(1-x^{j} y\right)-\left(1-x^{i} y\right)=\left(x^{i}-x^{j}\right) y .
$$

Hence, $x^{i+j}$ and $x^{i}-x^{j}$ commute with both $x$ and $y$ and therefore with each element in $R(A ; i, j, 1,1)$.

Lemma 2.9. If $\operatorname{gcd}(i, j)=d$, then there exists polynomials $p(x), q(x) \in A[x]$, both alternating sums of powers of $x$, such that $y x^{d}=p(x)+q(x) y$ in $R(A ; i, j, 1,1)$.

Proof. If $i=j$, then $\operatorname{gcd}(i, j)=i$ and we have $y x^{i}=1-x^{i} y$.

Now suppose $i \neq j$ and let $d=\operatorname{gcd}(i, j)$. Since $d=\operatorname{gcd}(i+j, j)$, there exist $m, n \in \mathbb{N}$ such that $d=n j-m(i+j)$, and so $y x^{n j}=y x^{m(i+j)+d}$. By Proposition 2.8, $x^{i+j}$ is in the center of $R(A ; i, j, 1,1)$ and so by Lemma 2.3 we obtain

$$
x^{m(i+j)} y x^{d}=y x^{m(i+j)+d}=y x^{n j}=(-1)^{n} x^{i n} y+\sum_{k=0}^{n-1}(-1)^{k} x^{(n-1) j+k(i-j)} .
$$

Now, by Corollary 2.7, the inverse of $x$ is a power of $x$ and hence $x^{r} x^{m(i+j)}=1$ for some $r$. Therefore, by (3) we have

$$
y x^{d}=(-1)^{n} x^{i n+r} y+\sum_{k=0}^{n-1}(-1)^{k} x^{(n-1) j+k(i-j)+r} .
$$

Theorem 2.10. If $i>j$ and $\operatorname{gcd}(i, j)=1$, then $R(A ; i, j, 1,1)$ is a finitely generated $A$-module with a generating set of cardinality at most $2(i+j-1)(i-j)$.

Proof. Since $\operatorname{gcd}(i, j)=1$, by Theorem 2.6 and Lemma 2.9, we have relations which work as reductions for $x^{n}$ and $y x$ respectively, for $n \geq(i+j-1)(i-j)$. Using these reductions, we can write every monomial/word of $x$ and $y$ in $R(A ; i, j, 1,1)$ as an $A$-linear combination of elements from the set

$$
\left\{1, x, x^{2}, \ldots, x^{(i+j-1)(i-j)-1}, y, x y, \ldots, x^{(i+j-1)(i-j)-1} y\right\}
$$

Hence, $R(A ; i, j, 1,1)$ is a finitely-generated $A$-module with generating set of cardinality at most $2(i+j-1)(i-j)$. 
2.2. Matrix descriptions. In this subsection we will obtain a complete description of the $A$-algebra $R(A ; i, j, 1,1)$ when $\operatorname{gcd}(i, j)=1$. Letting $n=2, a=x^{i}$, $b=x^{j}$, and $f=y$, then $\left\{E_{h k}: 1 \leq h, k \leq 2\right\}$, where $E_{h k}=y^{h-1} x^{i} y x^{j(k-1)}$, forms a set of $2 \times 2$ matrix units by Theorem 1.3 of [2] and so we have the following.

Observation 2.11. There exists an A-algebra $L$ such that $R(A ; i, j, 1,1) \cong M_{2}(L)$.

If we let $e_{h k}=E_{(3-h)(3-k)}$ for each $h$ and $k$, then it is easy to verify the $2^{4}+1=$ 17 relations from Theorem 1.1 to show that $\left\{e_{h k}: 1 \leq h, k \leq 2\right\}$ also forms a complete set of $2 \times 2$ matrix units, where $e_{11}=y x^{j}, e_{12}=y, e_{21}=x^{i} y x^{j}$, and $e_{22}=x^{i} y$.

By Observation 2.11 and the $2 \times 2$ matrix units $\left\{e_{h k}: 1 \leq h, k \leq 2\right\}$, we have an isomorphism $\phi: R(A ; i, j, 1,1) \rightarrow M_{2}(L)$. Identifying $R(A ; i, j, 1,1)$ with $M_{2}(L)$ via $\phi$, we have $y x^{j}=\left(\begin{array}{ll}1 & 0 \\ 0 & 0\end{array}\right) y=\left(\begin{array}{ll}0 & 1 \\ 0 & 0\end{array}\right), x^{i} y x^{j}=\left(\begin{array}{ll}0 & 0 \\ 1 & 0\end{array}\right)$ and $x^{i} y=\left(\begin{array}{ll}0 & 0 \\ 0 & 1\end{array}\right)$ since $y x^{j}=e_{11}, y=e_{12}, x^{i} y x^{j}=e_{21}$, and $x^{i} y=e_{22}$. Hence, we will now and for the rest of this subsection view $R(A ; i, j, 1,1)$ as the matrix ring $M_{2}(L)$. Letting $x^{j}=\left(\begin{array}{ll}a & b \\ c & d\end{array}\right)$ and $x^{i}=\left(\begin{array}{cc}p & q \\ s & t\end{array}\right)$ for some $a, b, c, d, p, q, s, t \in L$, we get from $y \cdot x^{i}=y x^{i}$ and $x^{i} \cdot y=x^{i} y$ the following matrix equations $\left(\begin{array}{ll}0 & 1 \\ 0 & 0\end{array}\right)\left(\begin{array}{ll}a & b \\ c & d\end{array}\right)=\left(\begin{array}{ll}1 & 0 \\ 0 & 0\end{array}\right)$ and $\left(\begin{array}{ll}p & q \\ s & t\end{array}\right)\left(\begin{array}{ll}0 & 1 \\ 0 & 0\end{array}\right)=\left(\begin{array}{ll}0 & 0 \\ 0 & 1\end{array}\right)$, and so $d=p=0$ and $c=s=1$. Since $x^{j} x^{i}=x^{i} x^{j}$, we also obtain $\left(\begin{array}{ll}a & b \\ 1 & 0\end{array}\right)\left(\begin{array}{ll}0 & q \\ 1 & t\end{array}\right)=\left(\begin{array}{ll}0 & q \\ 1 & t\end{array}\right)\left(\begin{array}{ll}a & b \\ 1 & 0\end{array}\right)$ and hence $q=b, t=-a, a q=-b t$, and so $a b=b a$. Further,

$$
x^{i+j}=\left(\begin{array}{ll}
b & 0 \\
0 & b
\end{array}\right) \text { and } x^{j}-x^{i}=\left(\begin{array}{cc}
a & 0 \\
0 & a
\end{array}\right),
$$

and so by Proposition 2.8 we have the following.

Claim 2.12. Here $a, b \in L$ are in the center of $L$ and thus $A[a, b] \subseteq L$.

Suppose now $\operatorname{gcd}(i, j)=1$. If $i>j$, then since $x$ is invertible by Theorem 2.5, then so is $x^{i+j}$ and also $b$ by Corollary 2.7 in $A[a, b]$. Further, there are $\alpha, \beta \in \mathbb{N}_{0}$ such that $1=\alpha j-\beta i$ and so

$$
x=x^{\alpha j-\beta i}=\left(\begin{array}{cc}
a & b \\
1 & 0
\end{array}\right)^{\alpha}\left(\begin{array}{cc}
0 & b \\
1 & -a
\end{array}\right)^{-\beta}=\left(\begin{array}{ll}
a & b \\
1 & 0
\end{array}\right)^{\alpha}\left(\begin{array}{ll}
a & b \\
1 & 0
\end{array}\right)^{\beta} \frac{1}{b^{\beta}} \in M_{2}(A[a, b]) .
$$

If $i=j$, then $i=j=1$ and hence $x=\left(\begin{array}{cc}a & b \\ 1 & 0\end{array}\right)=\left(\begin{array}{cc}0 & b \\ 1 & -a\end{array}\right)$ and so $a=0$ and $x=\left(\begin{array}{ll}0 & b \\ 1 & 0\end{array}\right) \in M_{2}(A[a, b])$. Therefore, if $\operatorname{gcd}(i, j)=1$, then in the isomorphism $R(A ; i, j, 1,1) \cong M_{2}(L)$ we see that both $x$ and $y$ are mapped to $M_{2}(A[a, b])$ and so all of $R(A ; i, j, 1,1)$ is mapped to $M_{2}(A[a, b])$. Therefore $M_{2}(L)=M_{2}(A[a, b])$ and so $L=A[a, b]$. This is summed up in the following proposition. 
Proposition 2.13. If $a$ and $b$ are as in Claim 2.12, $\operatorname{gcd}(i, j)=1$ and $i>j$ then $R(A ; i, j, 1,1) \cong M_{2}(A[a, b])$ as A-algebras. In particular, $L$ from Observation 2.11 is commutative.

Note that to obtain Proposition 2.13 we use the fact that the equality $M_{2}(L)=$ $M_{2}(A[a, b])$ implies $L=A[a, b]$. If the equality in $M_{2}(L)=M_{2}(A[a, b])$ is replaced by an isomorphism, we cannot draw the same conclusions: In the papers [11] and [6] examples of non-isomorphic, non-commutative rings $A$ and $B$ are given such that $M_{n}(A) \cong M_{n}(B)$. In fact, it is shown in [6] that there is an uncountable family of pairwise non-isomorphic rings $\left\{S_{\alpha}\right\}$ such that $M_{2}\left(S_{\alpha}\right) \cong M_{2}\left(S_{\beta}\right)$. Further, all $S_{\alpha}$ are Noetherian domains that are finitely-generated over their centers.

The rest of this subsection is devoted to the description of the commutative $A$ algebra $L=A[a, b]$ when we have $\operatorname{gcd}(i, j)=1$. Under this assumption we get by Lemma 2.9 a commuting reduction rule for $y x$ in $R(A ; i, j, 1,1)$. For this analysis we begin with a definition.

Definition 2.14. Let $A[s, t]$ be the polynomial ring in two variables $s$ and $t$ over $A$ and let $f: \mathbb{N}_{0} \rightarrow A[s, t]$ be defined recursively in the following way: $f(0)=0$, $f(1)=1$, and $f(n)=t f(n-1)+s f(n-2)$ for $n \geq 2$.

The following is easily obtained by induction on $n$.

Lemma 2.15. For $n \geq 1$, we have $\left(\begin{array}{cc}t & s \\ 1 & 0\end{array}\right)^{n}=\left(\begin{array}{cc}f(n+1) & s f(n) \\ f(n) & s f(n-1)\end{array}\right)$.

The main result of this section is the following theorem, in which a complete description of the algebra $A[a, b]$ from Proposition 2.13 is given.

Theorem 2.16. Let $f$ be as in Definition 2.14. If $\operatorname{gcd}(i, j)=1$, then we have $R(A ; i, j, 1,1) \cong M_{2}(A[s, t] / I)$ where

$$
I=\left(f(i+j), f(i+j-1)-s^{j-1}, s^{i-j}-(-1)^{i-j}\right)
$$

and $A[s, t]$ is the polynomial ring in two indeterminates $s$ and $t$ over $A$.

Before beginning our proof, we discuss some interesting consequences of Proposition 2.13 and Theorem 2.16.

First, for any ring $R$, the matrix ring $M_{n}(R)$ and $R$ are Morita equivalent (see [3]), meaning there is an equivalence of their modules in a categorical sense. By Theorem 2.16 we have in particular that $R(A ; i, j, 1,1)$ is Morita equivalent to a commutative ring when $\operatorname{gcd}(i, j)=1$.

For the second consequence, we recall that a polynomial identity ring (or a PI ring for short) $R$ is a ring such that there exists a polynomial in non-commuting 
indeterminates $p\left(x_{1}, x_{2}, \ldots, x_{n}\right) \in R\left\langle x_{1}, x_{2}, \ldots, x_{n}\right\rangle$ such that $p\left(r_{1}, r_{2}, \ldots, r_{n}\right)=0$ for all $r_{i} \in R$. For example, any commutative ring $R$ is a PI ring since it satisfies the identity $x y-y x=0$. Similarly, any $2 \times 2$ matrix ring over a commutative ring is also a PI ring since it satisfies the Hall identity $(x y-y x)^{2} z=z(x y-y x)^{2}$. In fact, any $n \times n$ matrix ring over a commutative ring satisfies the Amitsur-Levitzki identity $[10]$

$$
S_{2 n}\left(x_{1}, x_{2}, \ldots, x_{2 n}\right)=\sum_{\pi \in \operatorname{Sym}(2 n)} \operatorname{sgn}(\pi) x_{\pi(1)} x_{\pi(2)} \ldots x_{\pi(2 n)}=0 .
$$

Therefore, by Theorem $2.16 R(A ; i, j, 1,1)$ satisfies both the Hall identity and the Amitsur-Levitzki identity $S_{4}\left(x_{1}, x_{2}, x_{3}, x_{4}\right)$ and is therefore a PI ring when $\operatorname{gcd}(i, j)=1$.

We now delve into the proof of Theorem 2.16.

Proof of Theorem 2.16. First CASE: Suppose $i=j$, and so $i, j=1$. This case is special, in great part since $L$ from Observation 2.11 is here not a finitely generated $A$-module, unlike the case when $i>j$ (see Theorem 2.10.)

First we note that in this case $I=\left(f(2), f(1)-s^{0}, s^{0}-(-1)^{0}\right)=(t)$ and so $A[s, t] / I=A[s, t] /(t)=A[s]$, the polynomial ring over $A$ in one indeterminate $s$.

In the $A$-algebra $R(A ; 1,1,1,1)$ we can show that $\left\{y x=1-x y, y^{2}=0\right\}$ forms a complete set of reductions under the degree lexicographic order, or deglex order for short w.r.t. $x<y$ and hence $R(A ; 1,1,1,1)$ has a free $A$-module basis given by $\left\{1, x, x^{2}, \ldots, y, x y, x^{2} y, \ldots\right\}$. To see that $R(A ; 1,1,1,1)$ is isomorphic to the $2 \times 2$ matrix algebra over $A[s]$, we introduce the variable $s:=x^{2} \in R(A ; 1,1,1,1)$ and so $R(A ; 1,1,1,1)=k\left\langle x, y, s: y x=1-x y, y^{2}=0, x^{2}=s\right\rangle$. This yields the following set of reductions $\left\{y x=1-x y, y^{2}=0, x^{2}=s\right\}$ under the deglex order $s<x<y$, which is not complete as in the Diamond Lemma by Bergman from [4], since it contains two overlap ambiguities $y x^{2}$ and $x^{3}$. Resolving the first one we get

$$
y x^{2}=(y x) x=(1-x y) x=x-x y x=x-x(1-x y)=x^{2} y=s y,
$$

on one hand, and $y x^{2}=y\left(x^{2}\right)=y s$ on the other, and hence we obtain a new relation $s y=y s$. Resolving the second one we get $x^{3}=x\left(x^{2}\right)=x s$ on one hand, and $x^{3}=\left(x^{2}\right) x=s x$ on the other, and hence we obtain a new relation $s x=x s$. By adding these two new relations to our system of deglex reductions we obtain a complete set of reductions $\left\{y x=1-x y, y^{2}=0, x^{2}=s, x s=s x, y s=s y\right\}$ under the deglex order. Therefore $s$ is in the center of $R(A ; 1,1,1,1)$ and so $R(A ; 1,1,1,1)$ is an $A[s]$-algebra. By our complete set of reductions we see that $R(A ; 1,1,1,1)$ is a free $A[s]$-module with a basis consisting of $\{1, x, y, x y\}$ and hence of rank 4 over 
$A[s]$. Further, $R(A ; 1,1,1,1)$ has the set of $2 \times 2$ matrix units $e_{11}=1-x y, e_{12}=$ $y, e_{21}=x-s y, e_{22}=x y$, which shows $R(A ; 1,1,1,1) \cong M_{2}(L)$ for some $A[s]$ algebra $L$. Since both $R(A ; 1,1,1,1)$ and $M_{2}(L)$ have rank 4 over $A[s]$, we have $T=A[s]$ and hence $R(A ; 1,1,1,1) \cong M_{2}(A[s])$ as $A[s]$-algebras.

SECOND CASE: Suppose $i>j$. In this case $L$ is a finitely generated $A$-module by Theorem 2.10. Let $I=\left(f(i+j), f(i+j-1)-s^{j-1}, s^{i-j}-(-1)^{i-j}\right)$ be as stated in Theorem 2.16. We note that $s^{-1}$ exists in $A[s, t] / I$ and is given by $s^{-1}=$ $(-1)^{i-j} s^{i-j-1}$. Since $\operatorname{gcd}(i, j)=1$, there are $\alpha, \beta \in \mathbb{N}_{0}$ such that $\alpha j-\beta i=1$. Let $X, Y \in M_{2}(A[s, t] / I)$ be given by $X=\frac{1}{s^{\beta}}\left(\begin{array}{cc}t & s \\ 1 & 0\end{array}\right)^{\alpha+\beta}$ and $Y=\left(\begin{array}{ll}0 & 1 \\ 0 & 0\end{array}\right)$. By definition of $I$ we have in $A[s, t] / I$ that $s f(i+j)=f(i+j)=0, s f(i+j-1)=s \cdot s^{j-1}=s^{j}$ and $f(i+j+1)=t f(i+j)+s f(i+j-1)=s^{j}$ and hence in $M_{2}(A[s, t] / I)$ we have by Lemma 2.15 that

$$
\left(\begin{array}{ll}
t & s \\
1 & 0
\end{array}\right)^{i+j}=\left(\begin{array}{cc}
s^{j} & 0 \\
0 & s^{j}
\end{array}\right)
$$

and hence, since $\alpha j-\beta i=1$ we have $(\alpha+\beta) j=1+\beta(i+j)$ and so we get from (5) that

$$
X^{j}=\frac{1}{s^{\beta j}}\left(\begin{array}{ll}
t & s \\
1 & 0
\end{array}\right)^{(\alpha+\beta) j}=\frac{1}{s^{\beta j}}\left[\left(\begin{array}{ll}
t & s \\
1 & 0
\end{array}\right)^{i+j}\right]^{\beta}\left(\begin{array}{ll}
t & s \\
1 & 0
\end{array}\right)=\left(\begin{array}{ll}
t & s \\
1 & 0
\end{array}\right) .
$$

Similarly, since $\left(\begin{array}{cc}t & s \\ 1 & 0\end{array}\right)^{-1}=\frac{1}{s}\left(\begin{array}{cc}0 & s \\ 1 & -t\end{array}\right)$ and $(\alpha+\beta) i=\alpha(i+j)-1$ we get

$$
X^{i}=\frac{1}{s^{\beta i}}\left(\begin{array}{cc}
t & s \\
1 & 0
\end{array}\right)^{(\alpha+\beta) i}=\frac{1}{s^{\beta i}}\left(\begin{array}{cc}
s^{\alpha j} & 0 \\
0 & s^{\alpha j}
\end{array}\right) \frac{1}{s}\left(\begin{array}{cc}
0 & s \\
1 & -t
\end{array}\right)=\left(\begin{array}{cc}
0 & s \\
1 & -t
\end{array}\right) .
$$

Therefore we have $X^{i} Y+Y X^{j}=I$ and $Y^{2}=0$ in $M_{2}(A[s, t] / I)$ and hence there is a well-defined $A$-algebra homomorphism $R(A ; i, j, 1,1) \rightarrow M_{2}(A[s, t] / I)$ with $x \mapsto X$ and $y \mapsto Y$. For this map we further have $x^{i+j} \mapsto\left(\begin{array}{cc}s & 0 \\ 0 & s\end{array}\right)$ and $x^{j}-x^{i} \mapsto\left(\begin{array}{cc}t & 0 \\ 0 & t\end{array}\right)$, and so this homomorphism is a surjection. By (4) this homomorphism induces an $A$-algebra surjection $L=A[a, b] \rightarrow A[s, t] / I$ where $b \mapsto s$ and $a \mapsto t$ since $L$ is commutative. This means, in particular, that $t$ and $s$ satisfy any equations that $a$ and $b$ do in $L=A[a, b]$. It remains to show that $a$ and $b$ satisfy the same relations over $A$ that $s$ and $t$ do in $A[s, t] / I$.

Since Lemma 2.15 holds for arbitrary $s$ and $t$, then we get the same equations by replacing $s$ with $a$ and $t$ with $b$ and hence we have in $R(A ; i, j, 1,1) \cong M_{2}(A[a, b])$ on one hand that $\left(x^{j}\right)^{i+j}=\left(\begin{array}{ll}a & b \\ 1 & 0\end{array}\right)^{i+j}=\left(\begin{array}{cc}f(i+j+1) & b f(i+j) \\ f(i+j) & b f(i+j-1)\end{array}\right)$ and on the other hand we obtain $\left(x^{j}\right)^{i+j}=\left(x^{i+j}\right)^{j}=\left(\begin{array}{ll}b & 0 \\ 0 & b\end{array}\right)^{j}=\left(\begin{array}{cc}b^{j} & 0 \\ 0 & b^{j}\end{array}\right)$. Since by Lemma $2.5 x$ is invertible, then so is $b$ in $L$, and we then obtain from the above two equations that 
$f(i+j-1)=b^{j-1}$ and $f(i+j)=0$. We therefore get that $a$ and $b$ satisfy the same relations in $L=A[a, b]$ as $t$ and $s$ do in $I$ respectively and so $L \cong A[s, t] / I$ and hence $R(A ; i, j, 1,1) \cong M_{2}(A[s, t] / I)$ which completes our proof.

In [1], it is shown that for $A=k$ a field, $R(k ; i, j, 1,1)$ always maps to some $M_{N}(k)$ and is therefore non-zero. It remains to show that if $\operatorname{gcd}(i, j)=1$, then for any commutative ring $A$ we have $R(A ; i, j, 1,1) \neq\{0\}$. For that, we need a few technical results for the function $f$. The following lemma can be shown with simple induction arguments.

Lemma 2.17. As a polynomial of $t$, we have (I) $f(n)$ is monic with degree $n-1$, (II) $f(2 n)$ has no constant term, (III) $f(2 n+1)$ has constant term $s^{n}$.

Lemma 2.18. Let $\bar{f}$ be the image of $f$ under the map $A[s, t] \rightarrow A[t], s \mapsto-1$, so $\bar{f}(n)=t \bar{f}(n-1)-\bar{f}(n-2)$. In this case we have for each $n \geq 1$ :

$$
\begin{aligned}
\bar{f}(2 n-1) & =(\bar{f}(n)+\bar{f}(n-1))(\bar{f}(n)-\bar{f}(n-1)), \\
\bar{f}(2 n)-1 & =(\bar{f}(n+1)-\bar{f}(n))(\bar{f}(n)+\bar{f}(n-1)), \\
\bar{f}(2 n)+1 & =(\bar{f}(n+1)+\bar{f}(n))(\bar{f}(n)-\bar{f}(n-1)) .
\end{aligned}
$$

Proof. We first note that (6) is by Definition 2.14 clearly true for $n=1,2$. We proceed by induction on $n$. Suppose now

$$
\bar{f}(2 m-1)=(\bar{f}(m)+\bar{f}(m-1))(\bar{f}(m)-\bar{f}(m-1))=\bar{f}(m)^{2}-\bar{f}(m-1)^{2}
$$

for all $m \leq n$. Then, by the defining recursion, we get

$$
\begin{aligned}
\bar{f}(2 n+1) & =t \bar{f}(2 n)-\bar{f}(2 n-1) \\
& =t[t \bar{f}(2 n-1)-\bar{f}(2 n-2)]-\bar{f}(2 n-1) \\
& =t^{2} \bar{f}(2 n-1)-t \bar{f}(2 n-2)-\bar{f}(2 n-1) \\
& =t^{2} \bar{f}(2 n-1)-[\bar{f}(2 n-1)+\bar{f}(2 n-3)]-\bar{f}(2 n-1) \\
& =\left(t^{2}-2\right) \bar{f}(2 n-1)-\bar{f}(2 n-3) .
\end{aligned}
$$

Using the induction hypothesis for $\bar{f}(2 n-1)$ and $\bar{f}(2 n-3)$ and the defining recursion, we further get

$$
\begin{aligned}
\bar{f}(2 n+1)= & \left(t^{2}-2\right)\left[\bar{f}(n)^{2}-\bar{f}(n-1)^{2}\right]-\left[\bar{f}(n-1)^{2}-\bar{f}(n-2)^{2}\right] \\
= & \left(t^{2}-2\right)\left[(t \bar{f}(n-1)-\bar{f}(n-2))^{2}-\bar{f}(n-1)^{2}\right] \\
& -\left[\bar{f}(n-1)^{2}-\bar{f}(n-2)^{2}\right] \\
= & \left(t^{4}-3 t^{2}+1\right) \bar{f}(n-1)^{2}-\left(2 t^{3}-4 t\right) \bar{f}(n-1) \bar{f}(n-2) \\
& +\left(t^{2}-1\right) \bar{f}(n-2)^{2} .
\end{aligned}
$$


Again, using the defining recurrence for $\bar{f}(n+1)$ and $\bar{f}(n)$, we obtain

$$
\begin{aligned}
\bar{f}(n+1)^{2}-\bar{f}(n)^{2}= & (\bar{f}(n+1)+\bar{f}(n))(\bar{f}(n+1)-\bar{f}(n)) \\
= & ([t \bar{f}(n)-\bar{f}(n-1)]+\bar{f}(n))([t \bar{f}(n)-\bar{f}(n-1)]-\bar{f}(n)) \\
= & ((t+1) \bar{f}(n)-\bar{f}(n-1))((t-1) \bar{f}(n)-\bar{f}(n-1)) \\
= & ((t+1)[t \bar{f}(n-1)-\bar{f}(n-2)]-\bar{f}(n-1)) \\
& \cdot((t-1)[t \bar{f}(n-1)-\bar{f}(n-2)]-\bar{f}(n-1)) \\
= & \left(\left(t^{2}+t-1\right) \bar{f}(n-1)-(t+1) \bar{f}(n-2)\right) \\
& \cdot\left(\left(t^{2}-t-1\right) \bar{f}(n-1)-(t-1) \bar{f}(n-2)\right) \\
= & \left(t^{4}-3 t^{2}+1\right) \bar{f}(n-1)^{2}-\left(2 t^{3}-4 t\right) \bar{f}(n-1) \bar{f}(n-2) \\
& +\left(t^{2}-1\right) \bar{f}(n-2)^{2} .
\end{aligned}
$$

Hence, we obtain from the last two displayed relations

$$
\bar{f}(2 n+1)=\bar{f}(n+1)^{2}-\bar{f}(n)^{2}=(\bar{f}(n+1)+\bar{f}(n-1))(\bar{f}(n+1)-\bar{f}(n)),
$$

and thus (6) is proved by induction.

We will use induction to prove both (7) and (8) simultaneously. For $n=1$ we have $\bar{f}(2)=t, \bar{f}(1)=1$ and $\bar{f}(0)=0$ and so $\bar{f}(2)-1=t-1=(\bar{f}(2)-\bar{f}(1))(\bar{f}(1)+$ $\bar{f}(0))$ and $\bar{f}(2)+1=t+1=(\bar{f}(2)+\bar{f}(1))(\bar{f}(1)-\bar{f}(0))$. Suppose $\bar{f}(2 m)-1=(\bar{f}(m+$ $1)-\bar{f}(m))(\bar{f}(m)+\bar{f}(m-1))$ and $\bar{f}(2 m)+1=(\bar{f}(m+1)+\bar{f}(m))(\bar{f}(m)-\bar{f}(m-1))$ for all $m \leq n$. Using the defining recurrence, (6), and our induction hypothesis we get,

$$
\begin{aligned}
\bar{f}(2 n+2)= & t \bar{f}(2 n+1)-\bar{f}(2 n) \\
= & t(\bar{f}(n+1)-\bar{f}(n))(\bar{f}(n+1)+\bar{f}(n)) \\
& -[(\bar{f}(n+1)-\bar{f}(n))(\bar{f}(n)+\bar{f}(n-1))+1] \\
= & (\bar{f}(n+1)-\bar{f}(n))(t \bar{f}(n+1)+t \bar{f}(n)-\bar{f}(n)-\bar{f}(n-1))-1 \\
= & (\bar{f}(n+1)-\bar{f}(n))(\bar{f}(n+2)+\bar{f}(n+1))-1 .
\end{aligned}
$$

Thus $\bar{f}(2 n+2)+1=(\bar{f}(n+1)-\bar{f}(n))(\bar{f}(n+2)+\bar{f}(n+1))$. Similarly, $\bar{f}(2 n+2)-1=$ $(\bar{f}(n+1)+\bar{f}(n))(\bar{f}(n+2)-\bar{f}(n+1))$, which completes our proof.

We now argue directly that if $\operatorname{gcd}(i, j)=1$, then $R(A ; i, j, 1,1) \neq\{0\}$ for any commutative ring $A$.

Theorem 2.19. If $\operatorname{gcd}(i, j)=1$ and $I$ is as in Theorem 2.16, then $I \neq A[s, t]$ and thus $R \neq\{0\}$. 
Proof. First Case: Suppose $i+j$ is even. Let $A[s, t] \rightarrow A[t]$ be the evaluation such that $s \mapsto 1$. Then $\bar{I}=(\bar{f}(i+j-1)-1, \bar{f}(i+j), 0)$. By Lemma 2.17, we have that $\bar{f}(i+j-1)$ has constant term 1 and so both $\bar{f}(i+j-1)-1$ and $\bar{f}(i+j)$ have no constant term. Therefore $I \subseteq(t)$ and thus $\bar{I} \neq A[t]$. Hence $I \neq A[s, t]$ and so $R \neq\{0\}$.

SeCond CASE: Suppose $i+j$ is odd. Let $A[s, t] \rightarrow A[t]$ be the evaluation such that $s \mapsto-1$. Then $\bar{I}=\left(\bar{f}(i+j-1)-(-1)^{j-1}, \bar{f}(i+j), 0\right)$. Regardless of the parity of $j-1$, both $\bar{f}(i+j-1)-(-1)^{j-1}$ and $\bar{f}(i+j)$ are monic by Lemma 2.17 , and they share a common factor by Lemma 2.18, and thus $\bar{I} \neq A[t]$. Hence $I \neq A[s, t]$ and so $R \neq\{0\}$.

2.3. Examples. We conclude this section with two examples, the first of which is a consequence of Theorem 2.16 and is stated in the following corollary.

Corollary 2.20. Let $A$ be a commutative ring, then $R(A ; 2,1,1,1) \cong M_{2}(A)$.

Proof. We know $R(A ; 2,1,1,1) \cong M_{2}(A[s, t] / I)$ where

$$
I=\left(f(3), f(2)-s^{0}, s^{1}-(-1)^{1}\right)=\left(t^{2}+s, t-1, s+1\right)=(t-1, s+1)
$$

and so $A[s, t] / I \cong A$ and therefore $R(A ; 2,1,1,1) \cong M_{2}(A)$.

Example 2.21. We now consider the specific $\mathbb{Q}$-algebra $R(\mathbb{Q} ; 4,3,1,1)$. Again, using Theorem 2.16, we know $R(\mathbb{Q} ; 4,3,1,1) \cong M_{2}(A[s, t] / I)$ where

$$
\begin{aligned}
I & =\left(f(7), f(6)-s^{2}, s^{1}-(-1)^{1}\right) \\
& =\left(t^{6}+5 s t^{4}+6 s^{2} t^{2}+s^{3}, t^{5}+4 s t^{3}+3 s^{2} t-s^{2}, s+1\right) \\
& =\left(t^{3}-t^{2}-2 t+1, s+1\right),
\end{aligned}
$$

since $s$ and -1 are in the same coset and the $\operatorname{gcd}\left(t^{6}-5 t^{4}+6 t^{2}-1, t^{5}-4 t^{3}+3 t-1\right)=$ $t^{3}-t^{2}-2 t+1$. Since $t^{3}-t^{2}-2 t+1$ is irreducible over $\mathbb{Q}$, then $\mathbb{Q}[s, t] / I$ is a field extension of $\mathbb{Q}$ given by $\mathbb{Q}(\lambda)$ where $\lambda \in \mathbb{C}$ satisfies the polynomial equation $\lambda^{3}-\lambda^{2}-2 \lambda+1=0$.

While $R(\mathbb{Q} ; 4,3,1,1) \cong M_{2}(\mathbb{Q}(\lambda))$, we still have $(4,3,1,1) \notin \mathcal{A}_{\mathbb{Q}}$ since there is no non-trivial homomorphism that maps a field extension to its base field. This is the case since 1 must map to 1 and hence $\mathbb{Q}$ must map identically to $\mathbb{Q}$.

Further, we note that by Theorem 2.6 we have for $x \in R(\mathbb{Q} ; 4,3,1,1)$ that $x^{6}-x^{5}+x^{4}-x^{3}+x^{2}-x+1=0$ where $x^{6}-x^{5}+x^{4}-x^{3}+x^{2}-x+1$ is irreducible over $\mathbb{Q}$. Since each matrix over $\mathbb{Q}$ satisfies its characteristic polynomial, this means that the matrix ring $M_{6}(\mathbb{Q})$ is the smallest possible matrix ring that $R(\mathbb{Q} ; 4,3,1,1)$ can be mapped to non-trivially. On the other hand, since, every field extension is 
a vector space over its base ring and every element of a field extension acts linearly on that vector space by multiplication, then every field extension can be realized as set of matrices of dimension equal to the degree of the extension. Therefore, since $\lambda$ satisfies the polynomial equation $\lambda^{3}-\lambda^{2}-2 \lambda+1=0$ of degree three, we have that $\mathbb{Q}(\lambda)$ is isomorphic to a subring of $M_{3}(\mathbb{Q})$. This gives an explicit isomorphism of $M_{2}(\mathbb{Q}(\lambda))$ into a subring of $M_{2}\left(M_{3}(\mathbb{Q})\right)=M_{6}(\mathbb{Q})$. This means that the smallest $N$ in [1] for which there is a non-trivial $\mathbb{Q}$-algebra homomorphism $R(\mathbb{Q} ; 4,3,1,1) \rightarrow M_{N}(\mathbb{Q})$ is here $N=6$. We will see that this observation agrees with Theorem 3.8 in the following Section 3.

\section{Surjections onto matrix rings over base fields}

In the previous section we showed in Theorem 2.16 that if $\operatorname{gcd}(i, j)=1$, then $R(A ; i, j, 1,1) \cong M_{2}(A[s, t] / I)$. However, we also argued that just because we have $R(A ; i, j, 1,1) \cong M_{2}(L)$ for some commutative ring $L$, does not necessarily mean that $(i, j, 1,1) \in \mathcal{A}_{A}$ from Definition 1.6, as Example 2.21 in the previous Subsection 2.3 showed us. In this section, we will restrict our attention to $R(k ; i, j, 1,1)$ where $k$ is a field and investigate the set $\mathcal{A}_{k} \in \mathbb{N}^{4}$ for various fields $k$.

Note that $(i, j, 1,1) \in \mathcal{A}_{k}$ is equivalent to: "One can find nonzero $2 \times 2$ matrices $x, y \in M_{2}(k)$ satisfying $x^{i} y+y x^{j}=1$ and $y^{2}=0$." Clearly, if $x=\left(\begin{array}{ll}0 & 1 \\ 1 & 0\end{array}\right)$ and $y=\left(\begin{array}{ll}0 & 1 \\ 0 & 0\end{array}\right)$, then we have $x^{2 u+1} y+y x^{2 v+1}=1$ and $y^{2}=0$ for any integers $u, v$, and so we trivially have the following observations.

Observation 3.1. For any field $k$ and odd $i, j \in \mathbb{N}$, we have $(i, j, 1,1) \in \mathcal{A}_{k}$.

For a given field $k$, we would ideally like to determine exactly for which $i, j \in \mathbb{N}$ we have $(i, j, 1,1) \in \mathcal{A}_{k}$. As this question is too general to generate any interesting results, we will focus on the base fields $\mathbb{Q}$ and $\mathbb{Z}_{p}$ for prime numbers $p \geq 2$. By left-right symmetry (or stronger, by Theorem 2.2) we can assume $j \geq i$.

\subsection{Reducing to a four dimensional matrix algebra.}

Definition 3.2. Let $k$ be a field. For $a, b \in k$ define

$$
\begin{aligned}
S(k ; i, j, a, b) & =R(k ; i, j, 1,1) /\left(x^{2}-a x+b\right) \\
& =k\left\langle x, y: x^{i} y+y x^{j}=1, \quad y^{2}=x^{2}-a x+b=0\right\rangle .
\end{aligned}
$$

We have the following lemma:

Lemma 3.3. Either $S(k ; i, j, a, b)$ is trivial or $S(k ; i, j, a, b) \cong_{k} M_{2}(k)$ as $k$-algebras. 
Proof. Assume $S(k ; i, j, a, b)$ is nonzero. Then as an image of $R(k ; i, j, 1,1)$, it must be a nonzero $2 \times 2$ matrix algebra. By applying the rule $x^{2}=a x-b$ one gets the formulas:

$$
x^{i}=f_{i} x+g_{i}, \quad x^{j}=f_{j} x+g_{j},
$$

where $f_{l}, g_{l} \in k$. Clearly since $S(k ; i, j, a, b)$ is nonzero, $f_{i}, f_{j} \neq 0$ must hold. By putting these expressions into the equation $x^{i} y+y x^{j}=1$ and then isolating $y x$, one gets an expression of the form

$$
y x=-\frac{f_{i}}{f_{j}} x y-\frac{g_{i}+g_{j}}{f_{j}} x+\frac{1}{f_{j}}
$$

which gives a commuting rule for $x$ and $y$. Hence $S(k ; i, j, a, b)$ is generated by $x$ and $y$ satisfying:

$$
x^{2}=a x-b, \quad y^{2}=0, \quad y x=-\frac{f_{i}}{f_{j}} x y-\frac{g_{i}+g_{j}}{f_{j}} x+\frac{1}{f_{j}}
$$

which makes $S(k ; i, j, a, b)$ a nonzero $k$-algebra spanned by $\{1, x, y, x y\}$ and can therefore be at most 4-dimensional. As a $2 \times 2$ matrix algebra over $k$, it must be of dimension exactly 4 , and so it must be isomorphic to $M_{2}(k)$.

We will now examine the conditions that $i, j$ and $k$ must satisfy in order for $(i, j, 1,1) \in \mathcal{A}_{k}$. We have already seen in Observation 3.1 that if both $i$ and $j$ are odd then $(i, j, 1,1) \in \mathcal{A}_{k}$ for all field $k$, so we will therefore concentrate on other values of $i$ and $j$. We will no longer assume $\operatorname{gcd}(i, j)=1$.

We note that if $(i, j, 1,1) \in \mathcal{A}_{k}$ then, since $R(k ; i, j, 1,1)$ can be mapped onto $M_{2}(k)$ in which every element satisfies its second degree characteristic polynomial, there must be $a, b \in k$ such that $S(k ; i, j, a, b)$ is nonzero, in which case it is isomorphic to $M_{2}(k)$ as a $k$-algebra. It therefore is sufficient to find the conditions $i, j$ and $k$ must satisfy such that there are $a, b \in k$ which make $S(k ; i, j, a, b)$ nonzero. Now, if $a, b \in k$ and $F$ is an extension field of $k$, then $S(F ; i, j, a, b)=S(k ; i, j, a, b) \otimes_{k} F$, so $S(k ; i, j, a, b)$ is nonzero if and only if $S(F ; i, j, a, b)$ is nonzero.

Lemma 3.4. For $(i, j) \neq(1,1)$ we have the following: if $x^{2}-a x+b \in k[x]$ is inseparable with double root $r \in \bar{k}$, the algebraic closure of $k$, then: $S(k ; i, j, a, b)$ is nonzero if and only if $\operatorname{char}(k) \mid i+j, \operatorname{char}(k) \chi i$ and $r^{j-i}=-1$.

Proof. $S(k ; i, j, a, b)$ is nonzero if and only if $S(\bar{k} ; i, j, a, b)$ is nonzero, so we assume either one. Since $x^{2}-a x+b=(x-r)^{2} \in \bar{k}[x]$, by putting $z=x-r$ we get a new representation of $S(\bar{k} ; i, j, a, b)$ as

$$
\bar{k}\left\langle y, z:\left(i r^{i-1} z+r^{i}\right) y+y\left(j r^{j-1} z+r^{j}\right)=1, y^{2}=z^{2}=0\right\rangle .
$$


By multiplying the first equation by $y$ left and right, one gets $i r^{i-1} y z y=y=$ $j r^{j-1} y z y$. Since our algebra is nonzero we get:

$$
i r^{i-1}=j r^{j-1} \neq 0 .
$$

By multiplying the same equation by $z$ left and right we get:

$$
\left(r^{i}+r^{j}\right) z y=z-j r^{j-1} z y z, \quad\left(r^{i}+r^{j}\right) y z=z-i r^{i-1} z y z .
$$

By (9) we get $\left(r^{i}+r^{j}\right)(y z-z y)=0$. Since $S(\bar{k} ; i, j, a, b)$ is nonzero and hence $\cong_{k} M_{2}(k)$, it is noncommutative so $y z-z y \neq 0$, and so $r^{i}+r^{j}=0$ must hold. Since $(i, j) \neq(1,1)$ either $i-1$ or $j-1$ is greater than 0 so by $(9), r$ cannot be zero. We get therefore $r^{j-i}=-1$ and $j r^{j-i}=i \neq 0$, so we get the necessary conditions: $r^{j-i}=-1$ and $i+j$ is divisible by $\operatorname{char}(k)$ but neither $i$ nor $j$ are. These conditions are sufficient since if they hold, then one can map:

$$
y \mapsto\left(\begin{array}{cc}
0 & \frac{1}{i r^{i-1}} \\
0 & 0
\end{array}\right) z \mapsto\left(\begin{array}{ll}
0 & 0 \\
1 & 0
\end{array}\right) .
$$

Clearly these matrices satisfy the defining equations for $S(\bar{k} ; i, j, a, b)$ in its new representation.

Next we examine conditions that will make $S(k ; i, j, a, b)$ nonzero when $x^{2}-a x+$ $b \in k[x]$ is separable with two distinct roots $r, s \in \bar{k} . S(k ; i, j, a, b)$ is nonzero if and only if $S(\bar{k} ; i, j, a, b) \cong_{k} M_{2}(\bar{k})$. Now if the image of $x$ under this isomorphism satisfies $x^{2}-a x+b=(x-r)(x-s)$, then $x$ is mapped to a $2 \times 2$ matrix that has $(x-r)(x-s)$ as a minimal polynomial and is therefore diagonalizible with eigenvalues $r$ and $s$. We may therefore by an inner isomorphism of $M_{2}(\bar{k})$ assume the $\bar{k}$-algebra isomorphism $S(\bar{k} ; i, j, a, b) \cong M_{2}(\bar{k})$ to have the form $x \mapsto\left(\begin{array}{cc}r & 0 \\ 0 & s\end{array}\right)$ and $y \mapsto\left(\begin{array}{ll}b_{11} & b_{12} \\ b_{21} & b_{22}\end{array}\right)$. In order for these matrices to satisfy all the defining relations of $S(k ; i, j, a, b)$ we now only need to examine $x^{i} y+y x^{j}=1$ and $y^{2}=0$. The first equation gives the conditions:

$$
\left(r^{i}+r^{j}\right) b_{11}=1, \quad\left(r^{i}+s^{j}\right) b_{12}=0, \quad\left(s^{i}+s^{j}\right) b_{22}=1, \quad\left(r^{j}+s^{i}\right) b_{21}=0 .
$$

Clearly $r, s \neq 0$. Now if $b_{12}=0$ then, since $y^{2}=0$, we must have $b_{11}=b_{22}=0$ which is impossible. We have therefore $b_{12} \neq 0$. The same holds for $b_{21}$, so we have necessary conditions that $r$ and $s$ must satisfy:

$$
r^{i}+s^{j}=0 \neq r^{i}+r^{j}, \quad r^{j}+s^{i}=0 \neq s^{i}+s^{j} .
$$

If we do have $r, s \in k$ satisfying (10), then letting $b_{11}=\frac{1}{r^{i}+r^{j}}, b_{22}=\frac{1}{s^{i}+s^{j}}, b_{12}=$ $b_{21}=\frac{\mathbf{i}}{r^{i}+r^{j}}$, where $\mathbf{i}^{2}=-1$, it is easy to check that: $x \mapsto\left(\begin{array}{cc}r & 0 \\ 0 & s\end{array}\right), y \mapsto\left(\begin{array}{ll}b_{11} & b_{12} \\ b_{21} & b_{22}\end{array}\right)$ is 
indeed a $\bar{k}$-algebra isomorphism $S(\bar{k} ; i, j, a, b) \cong M_{2}(\bar{k})$. It is therefore sufficient to find $r, s \in \bar{k}$ satisfying (10).

Therefore, we are looking for $r, s \in \bar{k}$ satisfying a second degree polynomial over $k$, so in addition to the necessary conditions of (10) that $r, s \in \bar{k}$ must satisfy, we must also have $r+s, r s \in k$. Clearly these conditions combined are sufficient to make $S(k ; i, j, a, b)$ well defined and nonzero.

Lemma 3.5. For $r, s \in \bar{k}^{*}$, (10) are equivalent to:

$$
(r s)^{j-i}=1, \quad r^{i+j}+(r s)^{i}=0, r^{j-i} \neq-1 .
$$

So, $S(k ; i, j, a, b) \neq\{0\}$ if and only if there are $r, s \in \bar{k}^{*}$ satisfying (11) such that $r+s, r s \in k$.

By looking at the conditions of Lemma 3.5, along with the condition $r+s, r s \in k$, we see that whether there is a root $r$ of $x^{i+j}+\zeta^{i} \in k[x]$ in $\bar{k}$, where $\zeta$ is a root of $x^{j-i}-1$ in $k$, such that $r+\zeta / r \in k$ and $r^{j-i} \neq-1$, depends not only on the characteristic of $k$ but also on what kind of an extension field of the base fields $\left(\mathbb{Q}\right.$ or $\left.\mathbb{Z}_{p}\right) k$ is. Since by Definition 1.6 we have for all fields that $k_{1} \subseteq k_{2}$ implies that $\mathcal{A}_{k_{1}} \subseteq \mathcal{A}_{k_{2}}$ and $\mathcal{B}_{k_{1}} \subseteq \mathcal{B}_{k_{2}}$, it seems natural to study the initial element in the category of fields with a certain characteristic. Hence we will consider the cases $k=\mathbb{Q}, \mathbb{Z}_{p}$ for primes $p \geq 2$. Since $-1=1$ in $k=\mathbb{Z}_{2}$ we will dispatch that special case first.

Theorem 3.6. $(i, j, 1,1) \in \mathcal{A}_{\mathbb{Z}_{2}}$ if and only if

$$
(i, j) \equiv \begin{cases}(1,1) & (\bmod 2) \\ (1,2), & (2,1) \quad(\bmod 3) .\end{cases}
$$

Proof. We need to find necessary and sufficient conditions on $(i, j)$ such that one can find $a, b \in \mathbb{Z}_{2}$ with $S\left(\mathbb{Z}_{2} ; i, j, a, b\right)$ nonzero. There are two cases:

FIRST CASE: $x^{2}-a x+b$ is inseparable: Here by Lemma 3.4 we must have both $i$ and $j$ odd numbers, which is also sufficient by Observation 3.1.

SECOND CASE: $x^{2}-a x+b$ is separable: Here by Lemma 3.5 the roots must satisfy $r s=1, r^{i+j}=1, r^{j-i} \neq 1$ and $r+s \in \mathbb{Z}_{2}$ and so $r+1 / r=0$ or 1 .

If $r+1 / r=0$, then $r^{2}=1$ so $1=r^{i+j}=r^{j-i+2 i}=r^{j-i}$, which is impossible.

If $r+1 / r=1$, then $r^{2}+r+1=0$ and so $r^{3}=1$. Since $\operatorname{gcd}\left(x^{3}-1, x^{i+j}-1\right)=$ $x^{\operatorname{gcd}(3, i+j)}-1$, we must have $i+j$ divisible by 3 and $j-i$ not divisible by 3 . Since $i, j \equiv 0,1,2(\bmod 3)$, we must have $(i, j) \equiv(1,2)$ or $(2,1)(\bmod 3)$. This is also sufficient since $x \mapsto\left(\begin{array}{ll}0 & 1 \\ 1 & 1\end{array}\right), y \mapsto\left(\begin{array}{ll}0 & 1 \\ 0 & 0\end{array}\right)$ works for this case. 
3.2. The case for the field of rational numbers. Consider a class of polynomials $f_{n}(x) \in \mathbb{Z}[x]$ defined by:

$$
f_{0}(x)=2, \quad f_{1}(x)=x \text { and } f_{n+1}(x)=x f_{n}(x)-f_{n-1}(x), \text { for } n \geq 1 .
$$

By induction we easily get that

$$
x^{n}+x^{-n}=f_{n}\left(x+x^{-1}\right)
$$

for every $n \in \mathbb{N}$. If $k$ is a base field $\mathbb{Q}$ or $\mathbb{Z}_{p}$ then through the natural map $\mathbb{Z} \rightarrow k$ we can view the polynomials $f_{n}(x)$ in $k[x]$.

Suppose $k \neq \mathbb{Z}_{2}$ and there are $r, s \in \bar{k}$ and $n \in \mathbb{N}$ such that that $r+s \in k$, $r s \in k^{*}$ and $r^{2 n}+s^{2 n}=0$. Then by (13) we have $f_{n}(r / s+s / r)=\frac{r^{2 n}+s^{2 n}}{(r s)^{n}}=0$ and so $f_{n}(x)$ has a root $r / s+s / r \in k$. On the other hand if $f_{n}(x)$ has a root $t \in k$, then by (13) $t \neq-2$ and we can find $r, s \in \bar{k}$ with $r+s=r s=t+2 \in k$ and

$$
0=f_{n}(t)=f_{n}\left(\frac{(r+s)^{2}}{r s}-2\right)=f_{n}\left(\frac{r}{s}+\frac{s}{r}\right)=\frac{r^{2 n}+s^{2 s}}{(r s)^{n}} .
$$

Hence we have the following.

Lemma 3.7. For a base field $k$ there are $r, s \in \bar{k}$ such that $r+s \in k, r s \in k^{*}$ and $r^{2 n}+s^{2 n}=0$ if and only if $f_{n}(x)$ from (12) has a root in $k$.

We can now prove the main result of this subsection.

Theorem 3.8. $(i, j, 1,1) \in \mathcal{A}_{\mathbb{Q}}$ if and only if

$$
\begin{aligned}
(i, j) & \in\{(n, n) \mid n \not \equiv 0 \quad(\bmod 4)\} \text { or } \\
(i, j) & \equiv \begin{cases}(1,1) & (\bmod 2) \\
(1,2), & (2,1),(4,5),(5,4) \quad(\bmod 6) .\end{cases}
\end{aligned}
$$

Proof. To find out if there are $a, b \in \mathbb{Q}$ which will make $S(\mathbb{Q} ; i, j, a, b)$, nonzero we may by Lemma 3.4 assume $x^{2}-a x+b$ is separable with distinct roots $r, s \in \overline{\mathbb{Q}} \subseteq \mathbb{C}$. We now have two cases.

FIRST CASE: $i=j$ : Here the necessary and sufficient conditions for $S(\mathbb{Q} ; i, j, a, b)$ to be nonzero are, by Lemma 3.5, the existences of $r, s \in \mathbb{C}$ such that $r^{i}+s^{i}=$ $0, r+s \in \mathbb{Q}$ and $r s \in \mathbb{Q}^{*}$. Clearly if $i$ is odd one can let $r=1$ and $s=-1$, so assume $i=2 n$ to be even.

By Lemma 3.7 there are such $r, s \in \mathbb{C}$ if and only if $f_{n}(x)$ has a rational root, which is the case iff $f_{n}(x-2)$ has a rational root. By the recursive definition of $f_{n}$ in (12), we see that $f_{n}(x-2)$ is an $n$-th order polynomial with leading coefficient 1 and constant coefficient $(-1)^{n} 2$. So a rational number $a / b$ with $\operatorname{gcd}(a, b)=1$ is a 
root of $f_{n}(x-2)$ if and only if $b \mid 1$ and $a \mid 2$, hence the only possible rational roots of $f_{n}(x-2)$ are \pm 1 or \pm 2 . The only positive result one finds is $f_{n}(0)$ for which

$$
\left|f_{n}(0)\right|= \begin{cases}0 & \text { if } n \text { is odd } \\ 2 & \text { if } n \text { is even }\end{cases}
$$

holds. We therefore have from this and Observation 3.1 that $(i, i, 1,1) \in \mathcal{A}_{\mathbb{Q}}$ if and only if $i \not \equiv 0(\bmod 4)$.

SECOND CASE: $i \neq j$ : Again by left-right symmetry of $R(\mathbb{Q} ; i, j, 1,1)$ we may assume $j>i$. Here the necessary and sufficient conditions for $r, s \in \mathbb{C}$ to fulfill are by Lemma 3.5

$$
(r s)^{j-i}=1, r^{i+j}+(r s)^{i}=0, r^{j-i} \neq-1, r+s, r s \in \mathbb{Q} .
$$

Since $r s \in \mathbb{Q}$ and $(r s)^{j-i}=1$ we have $r s= \pm 1$. Since both $r$ and $s$ are here roots of unity in $\mathbb{C}$, then in order for $r+s \in \mathbb{Q}$ to hold, $s$ must be either $-r$ or $1 / r$.

If $s=-r$ then we get from (14) that $0=r^{2 i}\left(r^{j-i}+(-1)^{i}\right)$ and hence, $r^{j-i}=$ $(-1)^{i-1}$. Since $r^{j-i} \neq-1$ then $i$ must be odd. Also, $1=(-1)^{j-i} r^{2(j-i)}=(-1)^{j-i}$ and so $j$ must be odd as well. We conclude, what we already knew from Observation 3.1 , that $(i, j, 1,1) \in \mathcal{A}_{\mathbb{Q}}$ if both $i$ and $j$ are odd.

If $s=1 / r$ then (14) becomes

$$
r^{i+j}=-1, r+1 / r \in \mathbb{Q}, r^{j-i} \neq-1 .
$$

By looking at the two first equations of (15) one sees by (13) that $r+1 / r \in[2,2]$ is a rational root of $f_{i+j}(x)+2$. On the other hand if $c \in[2,2]$ is a rational root of $f_{i+j}(x)+2$ then by putting $r+1 / r=c$ one gets $r^{i+j}+r^{-(i+j)}=f_{i+j}(r+1 / r)=-2$ and so $r^{i+j}=-1$. So the existence of an $r$ in $\mathbb{C}$ satisfying the two first conditions of (15) is equivalent to the existence of a rational root $c \in[2,2]$ of $f_{i+j}(x)+2$.

The leading coefficient of $f_{n}(x)+2$ is 1 and the constant term is 2 if $n$ is odd, so the only possible rational roots of $f_{n}(x)+2$ in $[2,2]$ when $n$ is odd are \pm 1 or \pm 2 .

By (13) one gets $f_{2 n}(x)+2=f_{n}(x)^{2}$ so $f_{2 n}(x)+2$ has a rational root if and only if $f_{n}(x)$ has one. As we saw above $n$ must be odd and $f_{n}(0)=0$ is the only possibility.

We have therefore that the only possible rational roots of $f_{n}(x)+2$ in $[2,2]$ are $0, \pm 1, \pm 2$ and the only positive results we find are:

$$
\begin{array}{rlll}
f_{n}(0)+2=0 & \Leftrightarrow & n \equiv 2 & (\bmod 4) \\
f_{n}(1)+2=0 & \Leftrightarrow & n \equiv 3 & (\bmod 6) \\
f_{n}(-2)+2=0 & \Leftrightarrow & n \equiv 1 & (\bmod 2) .
\end{array}
$$


We have therefore the following cases for $r+1 / r$ being a root of $f_{i+j}(x)+2$ to consider:

$r+1 / r=0$ and $i+j=4 k+2$ : Here we have $r^{2}=-1$, so in order for $r^{j-i} \neq-1$ to hold we must have neither $i$ nor $j$ even and so we get no new information.

$r+1 / r=1$ and $i+j=6 k+3$ : Here we have $r^{2}-r+1=0$ and hence $r^{3}=1$, so in order for $r^{j-i} \neq-1$ to hold we must have neither $i$ nor $j$ divisible by 3 , hence $(i, j) \equiv(1,2),(2,1),(4,5)$ or $(5,4)(\bmod 6)$. It is on the other hand clear that for these values $(i, j)$ there is $r \in \mathbb{C}$ satisfying (15).

$r+1 / r=-2$ and $i+j$ odd: Here we have $r=-1$ and $j-i$ is odd so $r^{j-i}=-1$. This case is impossible, and we have completed the proof.

3.3. The case for a general prime number $p \geq 3$. For a prime number $p$ recall the $p$-adic order or the $p$-adic valuation of the integers $\nu_{p}: \mathbb{Z} \rightarrow \mathbb{N} \cup\{\infty\}$ defined by $\nu_{p}(n)=\max \left(\left\{\nu \in \mathbb{N}: p^{\nu} \mid n\right\}\right)$ for $n \neq 0$ and $\nu_{p}(0)=\infty$.

We now tackle the case $k=\mathbb{Z}_{p}$ for primes $p \geq 3$. We start with a few useful lemmas.

Lemma 3.9. If $G \subseteq k^{*}$ is a multiplicative subgroup of a field $k$ of characteristic $\neq 2$ and $|G|=n$, then $G$ has an element $g$ with $g^{m}=-1$ if and only if $\nu_{2}(m)+1 \leq \nu_{2}(n)$.

Proof. As a subgroup of $k^{*}$ then $G$ and so every subgroup of $G$ is cyclic. Let $\nu=\nu_{2}(m)$. If there is a $g \in G$ with $g^{m}=-1$, then $h^{2^{\nu}}=-1$ for some power $h$ of $g$. Since the characteristic is not 2 one has $h^{2^{\nu+1}}=1$ and $h^{2^{\nu}} \neq 1$. Hence $h$ has order $2^{\nu+1}$ in $G$ and so $2^{\nu+1}$ must divide $|G|$, the order of $G$.

If $2^{\nu+1}$ divides $|G|=n$, say $n=2^{\nu+1} c$, then let $g=\xi^{c}$ where $G=\langle\xi\rangle$. As an element of a field we have: $0=g^{2^{\nu+1}}-1=\left(g^{2^{\nu}}-1\right)\left(g^{2^{\nu}}+1\right)$ and hence $g^{2^{\nu}}=-1$ must hold and so $g \in G$ satisfies $g^{m}=-1$.

Lemma 3.10. For $c \in \mathbb{Z}_{p}$ and $z \in \overline{\mathbb{Z}}_{p}^{*}$ we have $z+\frac{c}{z} \in \mathbb{Z}_{p}$ if and only if $z^{p-1}=1$ or $z^{p+1}=c$.

Proof. For $t=z+\frac{c}{z} \in \mathbb{Z}_{p}$ we have $t \in \mathbb{Z}_{p}$ if and only if $t^{p}=t$, which again is equivalent to $\left(z^{p-1}-1\right)\left(z^{p+1}-c\right)=0$.

We can now dispatch the case when $i=j$.

Theorem 3.11. For a prime $p \geq 3$ we have $(i, i, 1,1) \in \mathcal{A}_{\mathbb{Z}_{p}}$ if and only if $\nu_{2}\left(p^{2}-\right.$ 1) $\geq \nu_{2}(i)+2$.

Proof. For an odd $i$ we have by Observation 3.1 that $(i, i, 1,1) \in \mathcal{A}_{\mathbb{Z}_{p}}$ and since $p \geq 3$ we have $\nu_{2}\left(p^{2}-1\right) \geq 2=\nu_{2}(i)+2$. Hence we can for the remainder of the proof assume $i$ to be even. 
We want to find $a, b \in \mathbb{Z}_{p}$ such that $S\left(\mathbb{Z}_{p} ; i, i, a, b\right)$ is nonzero. We have two cases.

FIRST CASE: $x^{2}-a x+b$ is inseparable: by Lemma 3.4 we see that if $i \neq 1$ then we must have that $p$ divides $2 i$ and not $i$, which is impossible since $p \geq 3$. Hence $i=1$ must hold which is covered in the theorem.

SECOND CASE: $x^{2}-a x+b$ is separable: assume the two roots are $r$ and $s$. By Lemma 3.5 the necessary and sufficient conditions for $S\left(\mathbb{Z}_{p} ; i, i, a, b\right)$ to be nonzero are the existences of $r, s \in \overline{\mathbb{Z}}_{p}$ such that:

$$
r^{i}+s^{i}=0, r+s \in \mathbb{Z}_{p}, r s \in \mathbb{Z}_{p}^{*}
$$

We will show that these conditions are equivalent to the existence of $\gamma \in \overline{\mathbb{Z}}_{p}$ such that:

$$
\gamma^{i}=-1 \text { and } \gamma+\gamma^{-1} \in \mathbb{Z}_{p} .
$$

That (16) implies (17) can clearly be gotten by putting $\gamma=r / s$. Then $\gamma^{i}+1=0$ and $\gamma+\gamma^{-1}=r / s+s / r=\frac{(r+s)^{2}}{r s}-2 \in \mathbb{Z}_{p}$.

The other implication we get by putting $r=\gamma+1$ and $s=\gamma^{-1}+1$. Then we have: $r^{i}+s^{i}=(\gamma+1)^{i}+\left(\gamma^{-1}+1\right)^{i}=\left(\gamma^{-1}+1\right)^{i}\left(\gamma^{i}+1\right)=0$ and $r+s=r s=\gamma+\gamma^{-1}+2 \in \mathbb{Z}_{p}$ where $i$ is even, $\gamma \neq-1$ and so $\gamma+\gamma^{-1}+2 \neq 0$, and therefore $r+s, r s \in \mathbb{Z}_{p}^{*}$.

It suffices therefore to find the conditions for $i$ and $p$ such that there exists a $\gamma \in \overline{\mathbb{Z}}_{p}$ satisfying (17).

By Lemma $3.10 \gamma+\gamma^{-1} \in \mathbb{Z}_{p}$ is equivalent to $\gamma$ satisfying $\left(\gamma^{p-1}-1\right)\left(\gamma^{p+1}-1\right)=0$, and so either $\gamma \in \mathbb{Z}_{p}^{*}$ or $\gamma \in G \subseteq \overline{\mathbb{Z}}_{p}^{*}$, where $G$ is the cyclic group of order $p+1$ formed by all the roots of $x^{p+1}-1 \in \mathbb{Z}_{p}[x]$.

In order for $\mathbb{Z}_{p}$ or $G$ to contain an element $\gamma$ such that $\gamma^{i}=-1$, it is by Lemma 3.9 necessary and sufficient that either $\nu_{2}(p-1) \geq \nu_{2}(i)+1$ or $\nu_{2}(p+1) \geq \nu_{2}(i)+1$. Since $\operatorname{gcd}(p-1, p+1)=2$, this is equivalent to $\nu_{2}\left(p^{2}-1\right) \geq \nu_{2}(i)+2$.

We will now conclude the article by finding necessary and sufficient conditions for $(i, j, 1,1) \in \mathcal{A}_{\mathbb{Z}_{p}}$ to hold for primes $p \geq 3$, together with a couple of corollaries. The proof is elementary and based on "case-chasing" using group theory and congruences.

Theorem 3.12. For a prime $p \geq 3$ we have $(i, j, 1,1) \in \mathcal{A}_{\mathbb{Z}_{p}}$ only in the following cases: (I) $\nu_{2}(j-i)>\nu_{2}(p-1) \geq \nu_{2}(i+j)$, (II) $\nu_{2}(j-i)=\nu_{2}(p-1) \neq \nu_{2}(i+j)$, (III) $\nu_{2}(j-i)<\nu_{2}(p-1)$ and $\operatorname{gcd}(j-i, p-1)$ is not an odd multiple of $\operatorname{gcd}(j+i, p-1)$, (IV) $\nu_{2}(j-i)<\nu_{2}(p-1), p \mid i+j$ and $p \nmid i,(V) \nu_{2}(j-i p)<\nu_{2}(p+1)+\min \left(\nu_{2}(j-\right.$ $\left.i), \nu_{2}(p-1)\right)$ and $j-i$ is not an odd multiple of $\operatorname{gcd}\left(j-i p,(p+1)(j-i), p^{2}-1\right)$. 
Remark 3.13. Note that in the case of $i=j$ we have $\nu_{2}(j-i)=\infty$ and so cases (II), (III) and (IV) do not occur. Case (I) reduces then to $\nu_{2}(p-1) \geq \nu_{2}(i)+1$ and case $(\mathrm{V})$ to $\nu_{2}(p+1) \geq \nu_{2}(i)+1$. We therefore see that Theorem 3.11 is covered by the above Theorem 3.12 .

Proof of Theorem 3.12. By Theorem 3.11 and by left-right symmetry of $R(k ; i, j, 1,1)$ we can assume $j>i$.

Convention: For convenience in writing this proof we define $d=\operatorname{gcd}(j-i, p-1)$ and $e=\operatorname{gcd}(j+i, p-1)$. Also, for the remainder of this proof we will be using $x, y$ and $z$ for integer variables in congruence equations and not as generators of our noncommutative algebra $R(A ; i, j, m, n)$.

To find out whether there are $a, b \in \mathbb{Z}_{p}$ with $S\left(\mathbb{Z}_{p} ; i, j, a, b\right)$ nonzero, we have, as in the proof of Theorem 3.11, two cases:

FIRST CASE: $x^{2}-a x+b$ is inseparable with a double root $r \in \mathbb{Z}_{p}$. If $r=0$ we must have $i=j=1$ which is not the case here, so $r \neq 0$. In this case by Lemma 3.4 we must have $p$ divide $i+j$ but not $i$ and $r^{j-i}=-1$ which by Lemma 3.9 can only occur when $\nu_{2}(j-i)<\nu_{2}(p-1)$ so we have gotten case $(I V)$ in the theorem.

SECOND CASE: $x^{2}-a x+b$ is separable with roots $r, s \in \overline{\mathbb{Z}}_{p}$. By Lemma $3.5 r$ and $s$ must satisfy the following conditions:

$$
(r s)^{j-i}=1, \quad r^{i+j}+(r s)^{i}=0, \quad r^{j-i} \neq-1, \quad r+s, r s \in \mathbb{Z}_{p} .
$$

Since $r s \in \mathbb{Z}_{p}$ here we have $(r s)^{p-1}=1$ and so if $d=\operatorname{gcd}(j-i, p-1)$, then $r s$ must be some power of a primitive $d$-th root of unity in $\mathbb{Z}_{p}$, that is $r s=\rho^{\beta y}$ where $\beta=\frac{p-1}{d}, y$ is some integer in $\mathbb{Z}$ and $\langle\rho\rangle=\mathbb{Z}_{p}^{*}$. So the conditions (18) become $r^{i+j}=-\rho^{\beta y i}, r^{j-i} \neq-1, r+\frac{\rho^{y \beta}}{r} \in \mathbb{Z}_{p}$. By Lemma $3.10 r+\frac{\rho^{y \beta}}{r} \in \mathbb{Z}_{p}$ is equivalent to $\left(r^{p-1}-1\right)\left(r^{p+1}-\rho^{y \beta}\right)=0$, so either $r \in \mathbb{Z}_{p}$, or $r \in \overline{\mathbb{Z}}_{p}$ satisfying $r^{p+1}=\rho^{y \beta}$ where $y \in \mathbb{Z}$ and so we have two sub-cases.

First SUB-CASE: $r \in \mathbb{Z}_{p}$. Since $r \neq 0$ we have that $r=\rho^{x}$ for some $x \in \mathbb{Z}$. So here we get the conditions

$$
\rho^{x(i+j)}=-\rho^{\beta y i}=\rho^{\frac{p-1}{2}+\beta y i} \text { and } \rho^{x(j-i)} \neq \rho^{\frac{p-1}{2}} .
$$

Since $\rho$ is the generator of $\mathbb{Z}_{p}^{*}$ it has period $p-1$ so we get the conditions for $x, y \in \mathbb{Z}$ :

$$
\begin{aligned}
x(i+j) & \equiv \frac{p-1}{2}+\beta y i \quad(\bmod p-1) \\
x(j-i) & \not \equiv \frac{p-1}{2} \quad(\bmod p-1)
\end{aligned}
$$


which is the same as whether one can find $x, y, z \in \mathbb{Z}$ such that:

$$
\begin{aligned}
2 x(i+j)-2 \beta y i & =(2 z+1)(p-1) \\
2 x(j-i) & \neq(\text { odd \# })(p-1) .
\end{aligned}
$$

Since $p-1=\beta d$, let $j-i=\alpha d$. Then $\operatorname{gcd}(\alpha, \beta)=1$ and $2 \beta i=\beta(i+j)-\alpha(p-1)$ and so we get:

$$
(i+j)(2 x-\beta y)=(2 z-\alpha y+1)(p-1), \quad 2 x(j-i) \neq(\operatorname{odd} \#)(p-1) .
$$

If $\nu_{2}(j-i)=\nu_{2}(p-1)$ then by definition, both $\alpha$ and $\beta$ are odd, say $\alpha=2 \alpha^{\prime}-1$ and $\beta=2 \beta^{\prime}-1$. Let us now change the variables $x$ and $z$ to $x^{\prime}=x-\beta^{\prime} y$ and $z^{\prime}=z-\alpha^{\prime} y$. Now the first equation of (19) becomes:

$$
(i+j)\left(2 x^{\prime}+y\right)=\left(2 z^{\prime}+y+1\right)(p-1)
$$

which clearly has a solution $x^{\prime}, y, z^{\prime} \in \mathbb{Z}$ and hence also $x, y, z \in \mathbb{Z}$ in this case, if and only if $\nu_{2}(i+j) \neq \nu_{2}(p-1)$, because $2 x^{\prime}+y$ and $2 z^{\prime}+y+1$ have distinct parity. When $\nu_{2}(j-i)=\nu_{2}(p-1) \neq \nu_{2}(i+j)$ then $2 x(j-i) \neq(\operatorname{odd} \#)(p-1)$ so we have gotten the case $(I I)$ in the theorem.

If $\nu_{2}(j-i)>\nu_{2}(p-1)$ then $\alpha$ is even and $\beta$ is odd, say $\alpha=2 \alpha^{\prime}$ and $\beta=2 \beta^{\prime}-1$. Again let $x^{\prime}=x-\beta^{\prime} y$ and $z^{\prime}=z-\alpha^{\prime} y$. Here the first equation of (19) becomes:

$$
(i+j)\left(2 x^{\prime}+y\right)=\left(2 z^{\prime}+1\right)(p-1)
$$

which clearly has solutions $x^{\prime}, y, z^{\prime} \in \mathbb{Z}$ and hence also $x, y, z \in \mathbb{Z}$ if and only if $\nu_{2}(p-1) \geq \nu_{2}(i+j)$. In this case $2 x(j-i)$ is never an odd multiple of $(p-1)$, so we have gotten here case $(I)$ in the theorem.

If $\nu_{2}(j-i)<\nu_{2}(p-1)$ then $\alpha$ is odd and $\beta$ is even, say $\alpha=2 \alpha^{\prime}-1$ and $\beta=2 \beta^{\prime}$. Let $x^{\prime}, z^{\prime}$ be as before and we get from (19) that

$$
(i+j)\left(2 x^{\prime}\right)=\left(2 z^{\prime}+y+1\right)(p-1) .
$$

Here we clearly can always find solutions $x^{\prime}, y, z^{\prime} \in \mathbb{Z}$ to (20) and hence also corresponding solutions in $x, y, z \in \mathbb{Z}$. Assume now that for every such solution $x, y, z \in \mathbb{Z}$ we have $2 x(j-i)=(\operatorname{odd} \#)(p-1)$. We first notice that this condition is equivalent to $x=($ odd \# $) \beta^{\prime}$, so we are here assuming that every solution $x, y, z \in \mathbb{Z}$ to $(i+j)(2 x-\beta y)=(2 z-\alpha y+1)(p-1)$ has $x=($ odd \# $) \beta^{\prime}$. Let us write $i+j=e t$ and $p-1=s e$ where $e=\operatorname{gcd}(i+j, p-1)$. Then $\operatorname{since} \operatorname{gcd}(s, t)=1$ every solution $x, y, z$ must satisfy:

$$
\begin{aligned}
2 x-\beta y & =\ell s \\
2 z-\alpha y+1 & =\ell t
\end{aligned}
$$


for some $\ell \in \mathbb{Z}$. Since $x^{\prime}=x-\beta^{\prime} y$ and $z^{\prime}=z-\alpha^{\prime} y$, then we assume that every solution $x^{\prime}, y, z^{\prime}$ to

$$
\begin{aligned}
2 x^{\prime} & =\ell s \\
2 z^{\prime}+y+1 & =\ell t
\end{aligned}
$$

has $x^{\prime}=($ odd $\#-y) \beta^{\prime}$, i.e. whenever $2 x^{\prime}=\ell s$ then $x^{\prime}=\left(\right.$ odd $\left.\#-\left(\ell t-2 z^{\prime}-1\right)\right) \beta^{\prime}=$ $\left(2 c_{\ell}-\ell t\right) \beta^{\prime}$ for some $c_{\ell} \in \mathbb{Z}$. So in particular when $x^{\prime}=s$ (i.e. $\ell=2$ ) we have $x^{\prime}=\left(2 c_{2}-2 t\right) \beta^{\prime}=\left(c_{2}-t\right) \beta$ and hence $s$ is even, which means $t$ is odd since $\operatorname{gcd}(s, t)=1$. We therefore have a solution $2 x^{\prime}=s$ (i.e. $\ell=1$ ) and from this we get $s=\left(2 c_{1}-t\right) \beta=($ odd $\#) \beta$, an odd multiple of $\beta$.

Suppose now that $s$ is an odd multiple of $\beta$, say $s=(2 w+1) \beta$ for some $w \in \mathbb{Z}$, then $x^{\prime}=\ell(2 w+1) \beta^{\prime}$ and $y=\ell t-\left(2 z^{\prime}+1\right)$. Since $t$ is odd, $\ell(2 w+1+t)-2 z^{\prime}$ is even and so $\ell(2 w+1)+y$ is odd, say $2 w^{\prime}+1$, and so $x^{\prime}=\ell(2 w+1) \beta^{\prime}=\left(2 w^{\prime}+1-y\right) \beta^{\prime}$. In summary, if $s$ is an odd multiple of $\beta$, then every solution $x^{\prime}, y, z^{\prime}$ to (20) has $x^{\prime}=($ odd $\#-y) \beta^{\prime}$.

We have therefore in the case $\nu_{2}(j-i)<\nu_{2}(p-1)$ that $s$ not being an odd multiple of $\beta$ is the necessary and sufficient condition, and since this is equivalent to $d \neq($ odd \# $) e$ we get case $(I I I)$ in the theorem.

SeCond Sub-CASE: $r^{p+1}=\rho^{y \beta}$, where $y \in \mathbb{Z}$. By (18) we want to find the conditions for the existence of $r \in \overline{\mathbb{Z}}_{p}$ such that:

$$
r^{i+j}=-\rho^{\beta y i}, r^{p+1}=\rho^{\beta y}, r^{j-i} \neq-1 .
$$

Since $r \neq 0$ and the cyclic group $\mathbb{Z}_{p}^{*}$ of order $p-1$ is generated by $\rho$, so $\mathbb{Z}_{p}^{*}=\langle\rho\rangle$, then (21) is equivalent to

$$
r^{j-i p}=-1, r^{(p+1) d}=1, r^{j-i} \neq-1 .
$$

The condition $r^{(p+1) d}=1$ is equivalent to $r \in G \subseteq \overline{\mathbb{Z}}_{p}^{*}$ where $G$ is the finite cyclic group of order $(p+1) d$ formed by all the roots of $x^{(p+1) d}-1$. By lemma 3.9 we must have $\nu_{2}((p+1) d)>\nu_{2}(j-i p)$.

Let us now assume that each solution of $r^{(p+1) d}=1, r^{j-i p}=-1$ satisfies $r^{j-i}=$ -1 . Let $\xi$ be the generator of $G$. Since $\xi$ is an element of $\overline{\mathbb{Z}}_{p}$, we have $0=$ $\xi^{(p+1) d}-1=\left(\xi^{\left(\frac{p+1}{2}\right) d}-1\right)\left(\xi^{\left(\frac{p+1}{2}\right) d}+1\right)$ so $\xi^{\left(\frac{p+1}{2}\right) d}=-1$. Since now $r$ is a power of $\xi$ we are in fact assuming that every $x$ satisfying $x(j-i p) \equiv\left(\frac{p+1}{2}\right) d$ $(\bmod (p+1) d)$ also satisfies $x(j-i) \equiv\left(\frac{p+1}{2}\right) d(\bmod (p+1) d)$. This is equivalent to assuming that every solution $x, y \in \mathbb{Z}$ to

$$
2 x(j-i p)=(2 y+1)(p+1) d
$$

has $2 x(j-i)$ as an odd multiple of $(p+1) d$. 
Since $\nu_{2}((p+1) d)>\nu_{2}(j-i p)$ we have $q=\operatorname{gcd}\left(j-i p, \frac{p+1}{2} d\right)=\operatorname{gcd}(j-i p,(p+$ 1)d). Write $j-i p=q u$ and $\frac{p+1}{2} d=q v$. Then every solution to (22) must be:

$$
\begin{aligned}
x & =\ell v \\
2 y+1 & =\ell u
\end{aligned}
$$

where $\ell \in \mathbb{Z}$. Clearly $\ell$ can only be odd. We have in particular for the solution $x=v$ and $y=\frac{u-1}{2}$ (i.e. $\left.\ell=1\right)$ that $2 x(j-i)=($ odd\# $) 2 q v$; that is to say $j-i=($ odd $\#) q$.

On the other hand, one can easily see that if $j-i=($ odd \# $) q$ then every solution to $(22)$ satisfies $2 x(j-i)=(\operatorname{odd} \#)(p+1) d$. We have therefore finally that one can find $r \in \overline{\mathbb{Z}}_{P}$ satisfying (21) if and only if $\nu_{2}((p+1) d)>\nu_{2}(j-i p)$ and $j-i \neq(\operatorname{odd} \#) \operatorname{gcd}(j-i p,(p+1) d)$. Since $\nu_{2}((p+1) d)>\nu_{2}(j-i p)=\nu_{2}(p+1)=$ $\min \left(\nu_{2}(j-i), \nu_{2}(p-1)\right)$ we finally have case $(V)$ in the theorem.

Remark 3.14. (i) The case that $i$ and $j$ are odd numbers is included in the theorem since $i, j$ both odd is the same as saying "Exactly one of the numbers $\nu_{2}(i+j), \nu_{2}(j-i) \in \mathbb{N}$ is equal to 1 and the other is 2 or greater".

(ii) The case $(V)$ in the theorem doesn't look symmetrical in $i, j$, but it is since $j-i p \equiv i-j p(\bmod (p+1) d)$ means that $\nu_{2}((p+1) d)>\nu_{2}(j-i p)$ if and only if $\nu_{2}((p+1) d)>\nu_{2}(i-j p)$.

We conclude the article by two corollaries of Theorem 3.12 for particular primes $p$. Since $\nu_{2}(p-1)=1$ is equivalent to $p \equiv-1(\bmod 4)$, we have by Theorem 3.12 and the Chinese Remainder Theorem the following corollary.

Corollary 3.15. For a prime $p$ of the form $p=2^{a}(2 b+1)-1$ where $a \geq 2$, in particular for each Mersenne prime of the form $p=2^{q}-1$ for a prime $q$, we have $(i, j, 1,1) \in \mathcal{A}_{\mathbb{Z}_{p}}$ only in the following cases: (I) $i$ and $j$ are both odd, (II) $i$ and $j$ have distinct parity and $\operatorname{gcd}(j-i, p-1)$ is not an odd multiple of $\operatorname{gcd}(j+i, p-1)$, (III)

$$
(i, j) \equiv(\ell(p+1),(p+1)(p-\ell)+p),((p+1)(p-\ell)+p, \ell(p+1)) \quad(\bmod 2 p),
$$

for $\ell \in\{1, \ldots, p-1\},(I V) \nu_{2}(j-i p) \leq a$ and $j-i$ is not an odd multiple of $\operatorname{gcd}\left(j-i p,(p+1)(j-i), p^{2}-1\right)$.

Further, for $p=3$ we note that the second condition (II) in the above Corollary 3.15 cannot occur, and since $p^{2}-1=8$, a pure power of 2 , the last condition (IV) becomes " $\nu_{2}(j-3 i) \leq 2$ and $j-i$ is not an odd multiple of $\operatorname{gcd}(j-3 i, 4(j-$ $i), 8)$." We now briefly translate this condition by considering two cases: (a) if 
$\nu_{2}(i) \neq \nu_{2}(j)$, then $i=2^{\nu} i^{\prime}$ and $j=2^{\nu} j^{\prime}$ where $i^{\prime}$ and $j^{\prime}$ have distinct parity and $\nu_{2}(j-i)=\nu_{2}(j-3 i)=\nu \leq 2$. Since both $j^{\prime}-i^{\prime}$ and $j^{\prime}-3 i^{\prime}$ are odd and $\operatorname{gcd}\left(j^{\prime}-3 i^{\prime}, 4\left(j^{\prime}-i^{\prime}\right), 2^{3-\nu}\right)=1$, then $j-i=2^{\nu}\left(j^{\prime}-i^{\prime}\right)$ is always an odd multiple of $2^{\nu} \operatorname{gcd}\left(j^{\prime}-3 i^{\prime}, 4\left(j^{\prime}-i^{\prime}\right), 2^{3-\nu}\right)=\operatorname{gcd}(j-3 i, 4(j-i), 8)$. (b) If $\nu_{2}(i)=\nu_{2}(j)=\nu$, then $i=2^{\nu}\left(2 i^{\prime}+1\right)$ and $j=2^{\nu}\left(2 j^{\prime}+1\right)$, and hence $j-i=2^{\nu+1}\left(j^{\prime}-i^{\prime}\right)$ and $j-3 i=2^{\nu+1}\left(j^{\prime}-3 i^{\prime}-1\right)$. By the first condition (I) we can assume $\nu \geq 1$, and since $\nu+\nu_{2}\left(j^{\prime}-3 i^{\prime}-1\right) \leq 1$ we can assume $\nu=1$ and $j^{\prime}-3 i^{\prime}-1$ to be odd. We note that $\operatorname{gcd}(j-3 i, 4(j-i), 8)=2^{\nu+1} \operatorname{gcd}\left(j^{\prime}-3 i^{\prime}-1,4\left(j^{\prime}-i^{\prime}\right), 2^{2-\nu}\right)=$ $2^{\nu+1} \operatorname{gcd}\left(j^{\prime}-3 i^{\prime}-1,2^{2-\nu}\right)$. Since $j^{\prime}-i^{\prime}$ and $j^{\prime}-3 i^{\prime}-1$ have distinct parity, $j^{\prime}-i^{\prime}$ is never an odd multiple of $\operatorname{gcd}\left(j^{\prime}-3 i^{\prime}-1,2^{2-\nu}\right)=1$. We therefore have from Corollary 3.15 the following.

Corollary 3.16. $(i, j, 1,1) \in \mathcal{A}_{\mathbb{Z}_{3}}$ if and only if

$$
(i, j) \equiv \begin{cases}(1,1) & (\bmod 2), \\ (1,2), & (2,1),(4,5),(5,4) \quad(\bmod 6), \\ (2,2), & (6,6) \quad(\bmod 8) .\end{cases}
$$

Acknowledgement. Sincere thanks to the anonymous referee for spotting some well-hidden typos and for improving the exposition of this article.

\section{References}

[1] G. Agnarsson, On a class of presentations of matrix algebras, Comm. Algebra, 24(14) (1996), 4331-4338.

[2] G. Agnarsson, S. A. Amitsur and J. C. Robson, Recognition of matrix rings II, Israel J. Math., 96(part A) (1996), 1-13.

[3] F. W. Anderson and K. R. Fuller, Rings and Categories of Modules, Second edition, Graduate Texts in Mathematics, 13, Springer-Verlag, New York, 1992.

[4] G. M. Bergman, The diamond lemma for ring theory, Adv. in Math., 29(2) (1978), 178-218.

[5] A. W. Chatters, Matrices, idealisers and integer quaternions, J. Algebra, 150(1) (1992), 45-56.

[6] A. W. Chatters, Nonisomorphic rings with isomorphic matrix rings, Proc. Edinburgh Math. Soc. (Ser. 2), 36(2) (1993), 339-348.

[7] T. Y. Lam, Lectures on Modules and Rings, Graduate Texts in Mathematics, 189. Springer-Verlag, New York, 1999.

[8] T. Y. Lam and A. Leroy, Recognition and computations of matrix rings, Israel J. Math., 96(part B) (1996), 379-397. 
[9] J. C. Robson, Recognition of matrix rings, Comm. Algebra, 19(7) (1991), 21132124.

[10] L. H. Rowen, Ring Theory: Student Edition, Academic Press, Inc., Boston, MA, 1991.

[11] S. P. Smith, An example of a ring Morita equivalent to the Weyl algebra $A_{1}$, J. Algebra, 73(2) (1981), 552-555.

Geir Agnarsson (Corresponding Author)

Department of Mathematical Sciences

George Mason University

MS 3F2

4400 University Drive

Fairfax, VA - 22030, USA

e-mail: geir@math.gmu.edu

Samuel S. Mendelson

The Naval Surface Warfare Center

Dahlgren Division

6149 Suite 203

Welsh Rd

Dahlgren, VA - 22448, USA

e-mail: samuel.mendelson@navy.mil 